

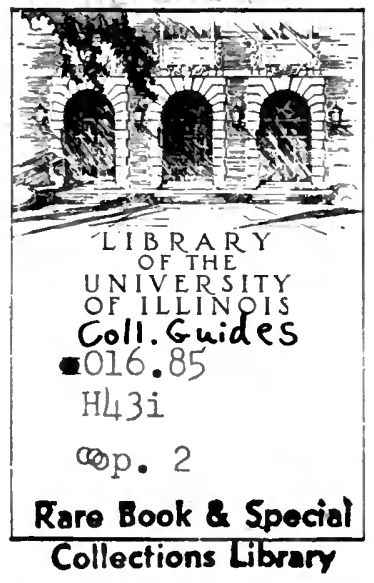



Italian Plays, 1500-1700, in the University of Illinois Library 



\section{Italian Plays, 1500-1700,}

in the
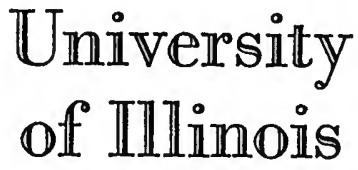

Lilbrary

Compiled by

Marvin T. Herrick 


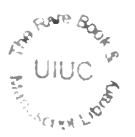

(C) 1966 by the Board of Trustees of the University of Illinois Manufactured in the United States of America Library of Congress Catalog Card No. 66-17248 

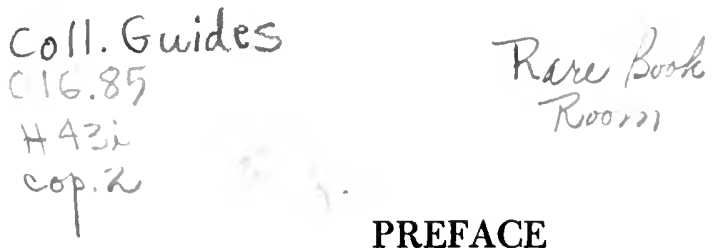

\section{PREFACE}

This collection is primarily of Italian plays by sixteenth-century authors. No special effort has been made to collect plays by seventeenth-century authors, but we have picked up a considerable number in the natural course of purchasing the older plays. Therefore I have extended the range to include plays in Italian published before 1701. No plays in Latin by Italian authors, of which we have a fair number, are included. The only exceptions to the rule are a few eighteenth-century printings when the printing is the first edition of a play written in the sixteenth century, when the printing is the only one we have, or when the printing is a reprint of an older edition that might prove useful for comparative study. I refer specifically to plays in Raccolta di commed. toscane [Napoli, 1720?] and in the Teatro comico fiorentino, 1750.

Several reference works have been useful for checking authors, titles, and printers, such as Lione Allacci's Drammaturgia, Venezia, 1755; Max Sander's Le Livre a figures italien, Milano, 1942; Nicolò Toppi's Biblioteca napoletana, Napoli, 1678; the British Museum General Catalogue of Printed Books; the Catalogue général des livres imprimés de la Bibliothèque Nationale.

The staff of the University Library in Urbana, from Robert Downs, the director, to the undergraduates who fetch books from the closed stacks, has gone out of its way, as usual, to be helpful. I am especially grateful to Isabelle Grant, Marian Harman, Suzanne Griffiths, Barbara Dalton, and David Langenberg of the Rare Book Room, and to Alma DeJordy and Helen Welch (Mrs. Preston Tuttle) of Acquisitions. 


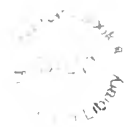




\section{ANONYMOUS PLAYS}

Aristippia: comedia. [Roma, 1524]. 5 acts; prose. -. [Vinegia, Nicolo d'Aristotile detto Zoppino], 1530. Ballo di donne turche insieme con i loro consorti di schiaui fatti libri. Danzato nel Real Pallazzo de Pitti dauanti alle Sereniss. Altezze di Toscana. Firenze, Cosimo Giunti, 1614. Verse.

- Comedia intitolata sine nomine. Fiorenza, i Giunti, 1574. 5 acts; prose.

Comedia noua volgare di uno eccellente poeta Mantuano. [Venetia, Jacobo Pentio da Leco, 1513]. Prose.

- Egloga rusticale di Grechoi, et del vescouo. Siena, [Antonio Mazochi, ad instantia di Maestro Giouanni d' Alexan[dro]], 1544. Colophon: In Siena per il faceto homo, F.S.D.S., ad istantia di un suo caro amico, 1542. Verse.

- La ghirlanda: giuoco pastorale. Rappresentato al Serenissimo Prencipe di Venetia, Marino Grimani. Alli 25. di Aprile, 1597. [n.p., n.d.]. Verse.

- Gratiana: favola boscareccia del Infiamato. Venetia, Giorgio Bizzardo, 1609. 5 acts; verse.

- L'Hortensio: comedia de gl' Academici Intronati. Siena, Luca Bonetti, 1576. 5 acts; prose. 
[Gl'ingannati]: comedia del Sacrificio degli Intronati celebrato nei giuochi dun carnovale in Siena. [n.p.], 1538. 5 acts; prose.

—. Vinetia, [Gioanne Padoano], [n.d.].

—. Vinegia, Agostino Bindoni, 1550.

-. Venetia, Plinio pietrasanta, 1554. In Girolamo Ruscelli's Delle comedie elette (1554).

-. Vinegia, Gabriel Giolito de' Ferrari, 1559.

—. Vinegia, Domenico Caualcalupo, 1585.

-. Venetia, Antonio Turini, 1609.

Glingannati: commedia. Siena, Matteo Florimi, [n.d.]. Lilia: egloga pastorale. [n.p., n.d.]. Verse.

Lilia: commedia pastorale. [Firenze, Agostin Simbeni, 1612].

Malfatto: roza e amorosa comedia da più Rozi composta. [Siena? Loggia del Papa? 1547?]. Incomplete: colophon missing.

- Siena, Loggia del Papa, 1614.

La moglie costante. Rappresentata al Serenissimo Prencipe di Venetia, Marino Grimani. Alli 20. di Maggio, 1599. Venetia, Gio. Antonio Rampazetto, [n.d.]. Verse.

Il mostro: favola pastorale. Rappresentata al Serenissimo Prencipe di Venetia, Marino Grimani. Alli 26. di Dicembre, 1596. [n.p., n.d.]. Verse.

Ottave alla Siciliana: contrastu bellissimo. Preposta, et riposta dell' Omo, e della Donna. Con altri strambotti alla Siciliana, nouamente posti in luce per me Paulo di Antonio di Palermo. Fiorenza, Gianantonio Caneo, 1595. Verse. 
Nuove ottave alla Ciciliana, con un' Dialogo d'un' Huomo, et una Donna: contrasto bellissimo. Et altre bellissime canzone, di nuouo composte per me Agostin Napolitano. Trevigi, Angelo Righettini, 1620. Verse.

Le nozze di Hadriana. Rappresentate al Serenissimo Prencipe di Venetia, Marino Grimani. Alli 26. di Dicembre, 1600. [n.p.], il Rampazetto, [n.d.]. Verse.

Pidinzuolo: commedia rusticale. Nuouamente composta: Per Tal di Tale: Ad instantia de Tali. [Siena, Michelagnolo di Bernardino Castagni, ad instantia di Giouanni di Alixandro, 1531]. Verse.

La Pippa: egloga rusticale. [Siena, Francesco di Simione, $\times 853$. $6420 \mathrm{p}$ e Compagni, 1547]. Verse.

Polifila: comedia. Fiorenza, [i Giunti], 1556. 5 acts; prose.

La rappresentatione del Agnolo Raffaello: e di Tobbia ${ }_{\times 853}$ Siena, [n.d.]. Verse.

La rappresentatione di Aman. [n.p., n.d.]. 5 acts; verse.

La rappresentazione di Costantino imperatore, e di San Siluestro Papa, e di Santa Helena imperatrice. [Firenze, OR 1588 Giouanni Baleni, 1588]. 2 parts; verse.

-. [Siena], [n.d.].

—. [Siena, Loggia del Papa], [n.d.].

La rappresentatione de' diecimila martiri, crocifissi nel Monte Arat, presso alla Città d' Alessandria: come riferisce San Girolamo, al tempo di Adriano, et Antonino Imperadori, l'anno del Signore 119, a di 22. di Giugnio. [Siena], [n.d.]. Verse.

La rappresentazione di Lazaro ricco: e di Lazaro pouero. [Siena, Loggia del Papa, 1610]. Verse. 
La rappresentatione e festa di Ottauiano Imperadore. [Siena, Loggia del Papa, 1613]. Verse.

La rappresentazione di Santa Agata Vergine, e Martire. [Firenze, Giouanni Baleni, 1591]. Verse.

. Siena, Loggia del Papa, 1606.

La rappresentazione di Santo Alesso. [Firenze, Giouanni Baleni, 1589]. Verse.

La rapresentatione di S.Antonio abate, il quale conuertì una sua sorella, e fecela monaca, e come non volendo tre ladroni accettare el suo consiglio sammazzorno l'un l'altro e furno portati a casa Satanasso, et egli fu terribilmente bastonato da i Diauoli. Siena, [n.d.]. Verse.

La devotissima rappresentatione di Santa Barbara. [Firenze, Giouanni Baleni, 1588]. Verse.

La rappresentazione di San Francesco. Quando conuerti tre Ladroni, che poi si fecion Frati. [Firenze, Giouanni Baleni, 1585]. Verse.

La rappresentatione di Santo Giorgio, Caualiere di Christo. [Siena], [n.d.]. Verse.

Rappresentazione di S. Grisante e Daria. [n.p., n.d.]. Verse. La rappresentatione e historia di Susanna. [Siena, Loggia del Papa, 1607]. Verse.

Rouinazo: comedia piaceuolissima da ridere de un Brauo chiamato Rouinazo. [n.p., n.d.]. Verse.

Tonio e Pippo contadini, e l'Hoste: commedia. [Firenze, Giouanni Baleni, 1588]. Verse.

Zannin da Bologna: frottola d'un padrone, et di uno servo. [Firenze, Zanobi Bisticci, 1604]. Verse. 
Abati, Antonio

I L'adoratione de' pastori al presepe di Christo: dramma musicale. Verse. In Poesie postume, Venetia, Angelo Bodio, 1673.

2 Il consiglio de gli dei: dramma da musica. 3 acts; verse. Separate t.p.; separate pagination. In Poesie postume.

3 Lo spedale: dramma burlesco. Verse. In Poesie postume.

Accolti, Bernardo

Virginia: comedia. [Firenze, 1518]. 5 acts; verse.

. [Vinegia, Nicolo di Aristotile detto Zoppino], 1535.

Alamanni, Luigi

6 Antigone: tragedia. No division into acts; verse. In Opere toscane, [Vineggia, Pietro di Nicolini da Sabbio, ad instantia di M. Marchio Sessa], 1533.

7 . In Opere toscane, Venetiis, Haeredes Lucae Antonii Iuntae, 1542. Colophon: Stampato in Vinegia per Pietro Sceffer Germano Maguntino, ad instantia delli heredi di M. Lucantonio Giunta.

8 La Flora: comedia. Firenze, Michelagnolo Sermartelli, 1601. 5 acts; verse.

\section{Aleardi, Lodovico}

9 Amida tiranno: tragedia. Vicenza, il Grossi, 1611. 5 acts; verse.

10 Il corsaro Arimante: fauola maritima. Vicenza, Lorenzo Lori, e Giacomo Cescato, 1610. Colophon: Nella Stam $\times 854$ AL25 OC peria di Francesco Grossi. 5 acts; verse. With Glauco 
schernito: fauoletta da recitarsi in Musica, per gl'Intermedij del Corsaro Arimante, del medisimo Auttore. 4 intermedii. Separate t.p.

Il corsaro Arimante: fauola maritima. Vicenza, Dominico Amadio, 1614.

\section{Allè, Girolamo}

Le chimere pitagoriche, cabaliste, chimiche et giudiciarie dissipate dal vento della verità: festa dotta, spirituale, curiosa, e rappresentabile, con tre intramezzi apparenti, due di Giobbe, et uno di Tobia. Bologna, Giacomo Monti, 1654. 3 acts; verse.

\section{Alticotio, Nicholo}

Pomona: commedia. [Siena, M[ichelangelo] di B[artolommeo] ad instantia di Giouanni di A[lexandro], 1524]. Verse.

\section{Ambra, Francesco d'}

I Bernardi: commedia. 5 acts; verse. In Teatro comico fiorentino (1750). A reprint of the 1564 edition.

La cofanaria: commedia. Con gl'Intermedi di Giovambatista Cini. 5 acts; verse. In Teatro comico fiorentino (1750). A reprint of the 1566 edition?

Il furto: comedia. Vinegia, Domenico Caualcalupo, 1584. 5 acts; prose.

16 . Venetia, Marc' Antonio Bonibelli, 1596.

ANDReini, Francesco

Le bravure del Capitano Spavento; divise in molti ragionamenti in forma di dialogo. Venetia, Giacomo Antonio Somasco, 1607. 55 dialogues; prose. 
Andreini, Grovanni Battista

L'Adamo: sacra rapresentatione. Milano, Geronimo Bordoni, 1617. 5 acts; verse.

ANgeli, Nicola degli

Arsinoe: tragedia. Venetia, Federico Abirelli, 1594. 5 acts; verse.

Angelio, Pietro

Edipo tiranno: tragedia di Sofocle. Ridotta dalla Greca nella Toscana lingua da M. Pietro Angelij Bargeo. Firenze, Bartolomeo Sermartelli, 1589. 5 acts; verse.

Anguillara, Giovanni Andrea dell'

Edippo: tragedia. Vinegia, Domenico Farri, 1565. 5 acts; verse.

Aperto Accademico Intronato (Belisario Bulgarini)

Gli scambi: commedia. Siena, Matteo Florimi, [n.d.]. 5 acts; prose. Separate t.p. In Commedie degl'Accademici Intronati, la seconda parte, Siena, ad instanza di Bartolomeo Franceschi, 1611.

Aragona, Tullia di

Tirrhenia: egloga del Mutio. Verse. In Rime della Signora Tullia di Aragona, Vinegia, Gabriel Giolito de Ferrari, 1549.

Aretino, Pietro

Quattro comedie. Il Marescalco, La cortegiana, La Talanta, L'hipocrito. [n.p.], 1588. All 5 acts; prose. Separate 
t.p.'s for all except Il Marescalco; continuous pagination. Bound with La terza, et ultima parte de ragionamenti, Gio. Andrea del Melagrano, [John Wolf, London], 1589.

Cortigiana: comedia. [n.p.], 1545.

Il fllosofo: comedia. Vinegia, Gabriel Giolito de Ferrari, 1549. 5 acts; prose.

Ariosto, Lodovico

Comedie. I suppositi, La cassaria, La Lena, Il negromante, $e$ La scolastica. Vinegia, Gabriel Giolito de' Ferrari, 1562. All 5 acts; verse. Separate t.p.'s for all except I suppositi. Cassaria: comedia. [Vinegia, Nicolo di Aristotile detto Zoppino], 1525. 5 acts; prose.

—. Cop. 2.

- [Vinegia, Marchio Sessa, 1536].

- [Vinegia, Nicolo di Aristotile di Ferrara detto Zoppino], 1538.

La Lena: comedia. [Vinegia, Nicolo d'Aristotile detto Zoppino], 1537. 5 acts; verse.

—. Vinegia, Gabriel Giolito de Ferrari e Fratelli, 1551.

Il negromante: comedia. [Vinegia, Nicolo d'Aristotile detto Zoppino], 1538. 5 acts; verse.

—. Vinegia, Gabriel Giolito de Ferrari, e Fratelli, 1551.

Scolastica: comedia. Vinegia, Gabriel Giolito de' Ferrari, 1562. 5 acts; verse. Same text as in Comedie (1562).

Gli soppositi: comedia. [Vinegia, Nicolo di Aristotile detto Zoppino], 1525. 5 acts; prose.

$\times 853$ An 4 OA Venetin, D Canalcelupo, 1587

8 
- [Vineggia, Francesco Bindoni e Mapheo Pasini compagni, 1537].

—. [Vineggia, Agustino de Bindoni, 1542].

Arsiccio Intronato (Antonio Vignali)

La Floria: comedia. Fiorenza, i Giunti, 1560. 3 acts; prose.

Fiorenza, i Giunti, 1567.

Avanzi, Giovanni Maria

Il Satiro: favola pastorale. Vinegia, Gio. Battista Sessa, e fratelli, 1587. Colophon: Vinegia, Giorgio Angelieri, ad instantia de gli Heredi del Sessa, 1587. 5 acts; verse.

Balsamino, Simone

Perla: tragicomedia. Venetia, Nicolò Moretti, 1596. 5 acts; verse.

. Cop. 2.

Bargagli, Girolamo

La pellegrina: commedia. Siena, Luca Bonetti, 1589. 5 acts; prose.

—. Siena, Matteo Florimi, [n.d.]. Separate t.p. In Commedie degl' Accademici Intronati, la seconda parte, ad instanza di Bartolomeo Franceschi, 1611.

Baroncini, Giuseppe

[Flaminio]: tragedia. Lucca, lo Busdrago, 1552. Only 2 acts marked; verse. 


\section{Bartolaia, Lodovico}

La ninfa cacciatrice: fauola boscareccia. Venetia, Alessandro de' Vecchi, 1620. 5 acts; verse.

\section{B[ARTOLOMMEI?], M[ATteO?] M[ARIA?]}

Amore, opera a caso: commedia. Firenze, et in Bologna, Gioseffo Longhi, [n.d.]. 3 acts; prose.

\section{Bartolommei Smeducci, Girolamo}

Tragedie. Eugenia: tragedia sacra; Isabella: tragedia sacra; Teodora: tragedia sacra; Giorgio: tragedia sacra; $\mathrm{Po-}$ lietto: tragedia sacra; Altamene: tragedia; Creso: tragedia. Roma, Francesco Cauallo, 1632. All 5 acts; verse.

- Impressione seconda. Firenze, Pietro Nesti, 1655. 2 vols. Includes all of the above plus Aglae: tragedia sacra; Clodoveo trionfante: tragedia sacra; Eustachio: tragedia sacra. All 5 acts; verse.

Bartolucci, Paolo Serenio

La Speranza: comedia. Con gli intermedi. Venetia, i Gioliti, 1587. 5 acts; prose.

Fra Baseo

Profezia: opera da recitare per frammesso a ueglie feste, conuiti e commedie, ridiculosa, piaceuole, onesta e bella. [n.p., n.d.]. Verse.

Basile, Giovanni Battista

Le avventurose disaventure: favola maritima. Venetia, Sebastiano Combi, 1612. 5 acts; verse. 
Begcari, Agostino

Il sacrificio: favola pastorale. Ferrara, ad instanza di Alfonso Caraffa, 1587. Facsimile: [Brescia, 1720]. 5 acts; verse.

[BeLCARI, FEO]

La rappresentazione di Abraam, et Isaac suo figliuolo. [Firenze, Giouanni Baleni, 1589]. Verse.

—. [n.p., n.d.].

La rappresentatione della Annuntiatione di nostra Donna. Siena, [n.d.]. Verse.

La rappresentazione di San Giouanni Batista quando andò nel diserto. [Firenze, Giouanni Baleni, 1589]. Verse.

—. Siena, Loggia del Papa, 1606.

La rappresentatione di Santo Panuntio Eremita: doue si rappresenta la conuersione d'uno, che era stato Ladrone di strada. Siena, Loggia del Papa, 1606. Verse.

\section{[Bellausa, Niccolò]}

La Flavia: comedia. [n.p., n.d.]. Lacks t.p. Allacci: "Trevigi, per Angiolo Mazzolini, 1590." 5 acts; prose.

Belo, Francesco

El Beco: comedia. [Roma, Antonio Blado d'Asula, 1538]. 5 acts; prose.

El pedante: comedia. [Roma, Valerio Dorico e Loygi fratelli Bresciani, 1538]. 5 acts; prose. 
Benedetti, Pietro

Il magico legato: tragicomedia pastorale. Anversa, Giouanni Keerbergio, 1607. Colophon: In Anversa. Appresso Andrea Bacx, 1606. 5 acts; verse.

Benedetto, Giovanna di

La fedeltà ingegnosa: comedia. Bologna, Gioseffo Longhi, 1687. 3 acts; prose. A prose version of an original verse drama.

Bentivoglio, Hercole

$\times 853$ B448F1545 I fantasmi: comedia. Vinegia, Gabriel Giolito de Ferrari, 1545. 5 acts; verse.

853 Bu40F1547 . Vinegia, Gabriel Giolito de Ferrari, 1547.

$\$ 853644861545$ Il geloso: comedia. Vinegia, Gabriel Giolito de Ferrari, 1545. 5 acts; verse.

Beolco, Angelo

See Ruzzante

Berni, Francesco

La Catrina: atto scenico rusticale. Insieme col frammesso detto il mogliazzo. [n.p., n.d.]. Verse. No t.p. Evidently an early eighteenth-century reprint of the 1567 edition. [

IL BibBiena

See Divitio

Bientina, IAcopo del

Fortuna: commedia. [Firenze, Giouanni Baleni, 1588]. Verse. 
Boccabadati, Giovanni Battista

Quando sta' peggio sta' meglio: opera scenica. Bologna, il Longhi, 1700. 3 acts; prose.

Boiardo, Matteo Maria

La commedia di Tozzo e Cappellina. Et il lamento di ser $\times 852863$ Cofaccia Buffone. [Firenze, Giouanni Baleni, 1587]. Verse.

Timone: comoedia traducta de uno dialogo de Luciano. [Scandiano, Peregrino di Pasquali e Gasparo Criuello, ${ }_{3630}^{\times 85} 0$ T500 1500]. 5 acts; verse.

Bonarelli della Rovere, Guidubaldo, conte

Filli di Sciro: favola pastorale. Venetia, Gio. Battista Ciotti, 1607. 5 acts; verse.

Bonarelli della Rovere, Prospero, conte

L'Imeneo: opera teotragicomica pastorale. Bologna, Ni- $\times 854$ colò Tebaldini, ad instanza de gli Eredi di Vangelista Dozza, 1641. 5 acts; verse.

Il Solimano: tragedia. [Firenze, Pietro Cecconcelli, 1620]. $\times 854285$ 5 acts; verse.

Bonifaccio, Baldassare

Amata: tragedia. Venetia, Antonio Pinelli, 1622. 5 acts; verse.

Bonifaccio, Gasparo

Amor venale: favola boschereccia. Venetia, Gio. Battista Ciotti, 1616. 5 acts; verse. 
Borghint, Raffaello

X854 B64938A L'amante furioso: comedia. Vinegia, Gio. Battista, e Gio. Bernardo Sessa, 1597. 5 acts; prose. 6 intermedii.

La donna costante: comedia. Fiorenza, Giorgio Marescotti, 1582. 5 acts; prose. 6 intermedii.

Bozza, Francesco

Fedra: tragedia. Vinegia, Gabriel Giolito de' Ferrari, 1578. 5 acts; verse.

Bracciolini, Francesco

$\times 854$ B 720 A L'amoroso sdegno: favola pastorale. Venetia, Gio. Battista Ciotti, 1597. 5 acts; verse.

\section{Venetia, Gio. Battista Ciotti, 1598.}

L'Evandro: tragedia. Seconda edizzione. Fiorenza, Giandonato, e Bernardino Giunti, e Compagni, 1613. 5 acts; verse.

X854 B720 P La Pentesilea: tragedia. Fiorenza, Gio. Donato, e Bernardino Giunti, e com., 1614. 5 acts; verse.

\section{Brina, Gabrielle}

Gl'imenei ravvivati: opera regia. Bologna, Giacomo Monti, 1666. 3 acts; prose.

Brunetto, Pier Giovanni

$\times 853$
B2348D1586 Dauid sconsolato: tragedia spirituale. Fiorenza, Giorgio Marescotti, 1586. 5 acts; verse.

Bruno, Grordano

Candelaio: comedia. Pariggi, Guglemo Giuliano, 1582. 5 acts; prose. 
Brusonio, Giovanni Giacomo

Comedia di mandata Sophia. Padoua, Giacomo Fabriano, 1550. 5 acts; prose.

Bulgarini, Belisario

See Aperto

Buonaparte, Niccolò

La vedoua: comedia. Fiorenza, i Giunti, 1568. 5 acts; $\times 853$ B8880V prose.

—. Fiorenza, Filippo Giunti, 1592. $\times 853 \quad 38980 \vee 1592$

Buonarotti, Michelagnolo, il Giovane

La Tancia: commedia rusticale. Firenze, Cosimo Giunti, $\begin{array}{r}\times 854 \\ 88801 / 612\end{array}$ 1612. 5 acts: verse.

- In Teatro comico fiorentino.

Buonfanti, Pietro

Errori incogniti: comedia. Firenze, Giorgio Marescotti, $\times 85488888$ 1586. Colophon: 1587. 5 acts; prose.

Cacciaconte, Ascanio

See Lo Strafalcione

Calcolona, Ettorre (Carlo Celano)

L'ardito vergognoso: [comedia]. Napoli, Antonio Bulifon, 1676. Colophon: In Napoli, per Michele Monaco, 1676. 5 acts; prose. 
Chi trionfa morendo, overo S. Casimiro. Napoli, Antonio Bulifon, 1676. Colophon: Per Michele Monaco, 1676. 3 acts; verse.

Galderari, Giovanni Battista

La Mora: comedia molto piacevole et sententiosa, ad imitatione de L'Eunuco di Terentio. [n.p.], 1594. 5 acts; prose. La schiava: comedia. Vicenza, Agostino dalla Noce, 1589. 5 acts; prose.

Calmo, Andrea (Scarpella Bergamasco)

4 La Fiorina: comedia. Vinegia, Iseppo Foresto, 1557. 3 acts; prose.

5 La potione: comedia. Vinegia, Stefano di Alessi, 1552. 4 acts; prose.

6 Rhodiana: comedia. Vinegia, Stephano di Alessi, 1553. 5 acts; prose. Ascribed to Ruzzante.

—. Vicenza, Domenico Amadio, 1617. Ascribed to Ruzzante.

Il Saltuzza: comedia. Vinegia, Stefano de Alessi, 1551. Colophon: In Vinegia appresso Batholomeo Cesana, 1551. 5 acts; prose.

La Spagnolas: comedia. Vinetia, Stephano di Alessi, 1554. 5 acts; prose.

Cammelli, Antonio

See Pistoia

Gampani, Niccolò (Lo Strascino)

10 Coltellino: comedia rusticale. Siena, Loggia del Papa, 1608. Verse. 
Magrino: comedia. [Fiorenza, nel Garbo, 1572]. Verse. Strascino: comedia rusticale. [Vineggia, Giovanmaria Pinardo, 1526]. Verse. With Maniscalco's Bicchiere.

[Siena, a S. Viglio a instantia di Giouanni di Alisandro, 1546]. With Trionfo di Pan.

CAmpeggi, Ridolfo, conte

Filarmindo: favola pastorale. [Bologna, gli Heredi di Gio. Rossi, 1605]. 5 acts; verse.

Il Tancredi: tragedia. [Bologna, Bartolomeo Cochi, 1614]. 5 acts; verse.

Campeli, Bernardino

Albesinda: tragedia. Venetia, Cristofforo Tomasini, 1623. 5 acts; verse.

Campiglia, Maddalena

Flori: favola boscareccia. Vicenza, Gl'heredi di Perin Libraro, e Tomaso Brunelli compagni, 1588. 5 acts; verse.

Canali, Francesco

L'Andreuccio del Boccaccio ridotto al rappresentabile: [commedia]. Vicenza, il Brescia, 1612. 3 acts; verse.

Carbone, Nicolò

Altea: tragedia. [Napoli, Mattio Cancer, 1559]. 5 acts; verse.

Gli amorosi inganni: comedia. Napoli, Raymondo Amato, [1559]. 5 acts; prose. 
Garo, Annibal

Gli straccioni: comedia. Vinegia, 1582. Aldine anchor on t.p. 5 acts; prose.

—. Venetia, Aldo, 1589.

Carretto, Galeotto dal

Noze de Psyche e Cupidine: [comedia]. [Milano, Augustino de Vicomercato ad instantia de Meser Ioanne Iacobo e fratelli de Legnano, 1520]. Verse.

La Sophonisba: tragedia. Vinegia, Gabriel Giolito de Ferrari, 1546. No division into acts; verse.

Tempio de amore: comedia. [Venetia, Nicolo Zopino e Vicentio compagno, 1524]. Verse.

- [Bologna, gli Heredi di Benedetto gia di Hettorre di Faelli, 1525].

Cartaio, Antonio Maria

See Lo Stechito

Casalio, Grovanni Battista

Amaranta: comedia nuova pastorale. Vinegia, [Nicolo d'Aristotele detto Zopino], 1538. 5 acts; verse.

\section{[Castalio, SABba]}

Il lamento pietoso del disgratiato Glonico pastore contra D'Amore e di Delia crudele da lui sommamente amata. [Vinegia, Gieronymo Penico da Lecco, 1528]. Verse. On Aii: "Incomincia la Barona composta per Sabba Castallio." Allacci (p. 137) gives Barona as title. 
Castelletti, Christoforo

L'Amarilli: pastorale. Venetia, Bartholomio Carampello, 1600. 5 acts; verse.

I torti amorosi: comedia. Venetia, Gio. Battista Sessa e Fratelli, 1581. 5 acts; prose.

\section{Castellini, Iacopo}

Asdrubale: tragedia. Fiorenza, L[orenzo] Torrent[ino], 1562. 5 acts; verse.

Il medico: comedia. Fiorenza, L. Torrentino, 1562. 5 acts; verse.

Cavalca, T. M.

See Valacca da Sarope

Cecchi, Giovanni Maria

Comedie. Libro primo. La dote, La moglie, Il corredo, La stiaua, Il donzello, $G l^{\prime}$ incantesimi, Lo spirito. Venetia, Bernardo Giunti, 1585. All 5 acts; verse. Separate t.p.'s for all except La dote.

\section{Cop. 2.}

Another issue with slight variations of main t.p.

L'assiuolo: comedia. Vinegia, Gabriel Giolito de Ferrari, e Fratelli, 1550. Colophon: 1551. 5 acts; prose.

I dissimili: comedia. Vinegia, Gabriel Giolito de Ferrari e Fratelli, 1550. 5 acts; prose.

Il donzello: comedia. Venetia, Bernardo Giunti, 1585. Same text as in Comedie. 
L'esaltazione della croce, con $i$ suoi intermedi. Firenze, Bartolomeo Sermartelli, 1589. 5 acts; verse. 6 intermedii.

—. Firenze, Michelagnolo di Bart. Sermartelli, 1592. Colophon: Nella Stamperia di Bartolomeo Sermartelli, l'Anno 1586.

Glincantesimi: comedia. Vinegia, Gabriel Giolito de Ferrari e Fratelli, 1550. 5 acts; prose.

La moglie: comedia. Vinegia, Gabriel Giolito de' Ferrari, et Fratelli, 1556. 5 acts; prose.

- Venetia, Bernardo Giunti, 1585. Same text as in Comedie.

Il servigiale: comedia. Con gli intermedii. Fiorenza, i Giunti, 1561. Colophon: In Fiorenza, appresso gli heredi di Bernardo Giunti, 1561. 5 acts; verse. 5 intermedii.

La stiaua: comedia. Vinegia, Gabriel Giolito de Ferrari e Fratelli, 1550. 5 acts; prose.

Celano, Carlo

See Calcolona

Cenci, Gracomo

Gli errori: comedia. [Vinegia, Cornelio de Nicolini da Sabbio a instantia de Marchio Sessa], [n.d.]. 5 acts; prose.

Cenni, Angiolo

See Il Resoluto

Cesana, Gasparo

La prova amorosa: favola pastorale. Venetia, Francesco Ciotti, 1606. 5 acts; verse. 


\section{Cesari, Cesare de'}

Cleopatra: tragedia. Venetia, Giouan. Griffio, 1552. $5 \times 853<3<8 \mathrm{C}$ acts; verse.

Romilda: tragedia. [Venetia, Francesco Bindoni, et Ma- $\times 853633$ ef pheo Pasini, 1551]. 5 acts; verse.

Scilla: tragedia. Venetia, Giouan. Griffio, 1552. 5 acts; $\times 85363305$ verse.

Ghaggio, Paolo

Flamminia prudente: novelletta [comedia]. Vinegia, al segno del Pozzo, 1551. 2 acts; prose.

\section{Chiabrera, Gabriello}

Gelopea: favola boschereccia. Venetia, Sebastiano Combi, 1607. 5 acts; verse.

Combi, 1610.

Meganira: favola boschereccia. 5 acts; verse. In Alcune poesie boschereccie di Gabriello Chiabrera, Fiorenza, Gio. Antonio Caneo, 1608.

-. Venetia, Sebastiano Combi, 1609.

-. In his Rime, Parte Terza.

Il rapimento di Cefalo rappresentato nelle nozze della cristianissima Maria Medici, Regina di Francia e di Nauarra. Firenze, Giorgio Marescotti, 1600. Quarto. 5 acts; verse. Il rapimento di Cefalo rappresentato nelle nozze della christianissima Regina di Francia e di Nauarra, Maria Medici. Firenze, Giorgio Marescotti, 1600. Octavo. 
Il rapimento di Cefalo. Venetia, Sebastiano Combi, 1605. Separate t.p. In Rime, Venetia, Sebastiano Combi, 1605.

\section{Crcognini, Giacinto Andrea}

L'Adamira ouero La statua dell'honore: opera scenica. Venetia, Nicolò Pezzana, 1662. 3 acts; prose.

L'amore tra nemici: opera comica. Venetia, Nicolò Pezzana, 1662. 5 acts; prose.

La donna più sagace fra l'altre: opera. Venetia, Nicolò Pezzana, 1662. 3 acts; prose. . Venetia, 1663.

La forza dell'amicitia, ouero L'honorato ruffiano di sua moglie: opera scenica. Venetia, Nicolò Pezzana, 1661. 3 acts; prose.

La forza del fato, overo Il matrimonio nella morte: opera tragica di lieto fine. Venetia, Nicolò Pezzana, 1662. 3 acts; prose.

- Bologna, gli H.H. di Domenico Barbieri, 1663.

La forza dell' innocenza ne' successi di Papirio: opera tragica. Venetia, Christofolo Ambrosini, 1672. Colophon: Corretta dal Dottore Francesco Maria Ambroni Correttorre approuato del Publico. 3 acts; prose.

Le gelosie fortunate del prencipe Rodrigo: opera. Venetia, Christofolo Ambrosini, 1672. 3 acts; prose.

Le glorie e gli amore di Alessandro Magno, e di Rossane: opera tragicomica. Venetia, Nicolò Pezzana, 1661. 3 acts; prose.

L'honorata poverta di Rinaldo: opera scenica. Venetia, [n.d.]. 3 acts; prose. 
La Mariene ouero il maggior mostro del mondo: [opera tragica]. Venetia, Zaccaria Conzatti, 1668. 3 acts; prose. Il marito delle due moglie: [opera scenica]. Venetia, Nicolò Pezzana, 1662. 3 acts; prose.

La moglie di quattro mariti: opera tragica. Venetia, Nicolò Pezzana, 1661. 3 acts; prose.

Santa Maria Egizziaca: [opera]. Venetia, Nicolò Pezzana, 1663. 3 acts; prose.

Cini, Desmerio

Desiderio, e Speranza fantastichi: comedia tropologica. Venetia, Sebastiano de Combi, 1607. 5 acts; prose.

—. Cop. 2. T.p. missing.

\section{Cini, Giacinto}

Il rosario trionfante: dramma spirituale. Padoua, Giulio $\times 854<44295$ Criuellari, ad instanza di Vicenzo Coradini, 1642. 5 acts; verse.

\section{[Claudi, Francesco]}

Le lettere di cambio: comedia de gl' ill. signori Acamedici Sviati, rappresentata in Siena. Venetia, Giouanni Alberti, 1606. 5 acts; prose.

Clemoneo, Erilo (Alessandro Guidi)

L'Endimione: [favola pastorale]. Roma, Gio. Giacomo Komarek Boemo, 1692. 5 acts; verse.

Collenucci[o], Pandolfo

Ioseph: comedia. Venetijs, 1564. 6 acts; verse. 
COMPARINI, LORENZO

Due comedie, ciò̀ Il pellegrino, et Il ladro. Vinegia, Gabriel Giolito de Ferrari et Fratelli, 1554. Both 5 acts; prose. Il ladro has separate t.p.

Contarini, Francesco

La finta fiammetta: fauola pastorale. Venetia, Ambrosio Dei, 1611. 5 acts; verse.

Intermedi rappresentati nella Finta Fiammetta in Padoa. Venetia, Ambrogio Dei, 1611. 4 intermedii; verse.

Contile, Luga

La Agia: egloga. [Mediolani, Valerius, et Hireronymus Metii, 1552]. Verse.

La Cesarea Gonzaga: comedia. [Milano, Francesco Marchesino, 1550]. 5 acts; prose.

La Nice: [poema drammatico]. [Milano, Valerio, e Girolamo fratelli da Meda, 1551]. Verse.

La Pescara: comedia. [Milano, Francesco Marchesino, 1550]. 5 acts; prose.

La trinozzia: comedia. [Milano, Francesco Marchesino, 1550]. 5 acts; prose.

Contrini, Francesco di Iacopo

Lite amorosa: egloga nuova. [n.p., n.d.]. Verse.

Cornacchini, Domenico

$\times 854$ C 140 \& La nativita di N.S. Giesu Cristo: rappresentazione. Fiorenza, Bartolommeo Sermartelli, 1607. 5 acts; verse. 
Cortese, Giulio Cesare

La Rosa: favola [boscareccia]. [n.p., n.d.]. Dedication at Naples, 1621. 5 acts; verse.

… In Opere, Napoli, ad instanza d' Adriano Scultore, 1666.

Cortesi, Cortese

Giustina Reina di Padoua: tragedia. Vicenza, Pietro Greco, e Giacomo Cescato Compagni. Ad istanza di Francesco Bolzetta. 1607. 5 acts; verse.

Cremonini, Cesare

Il nascimento di Venetia: poema [drammatico]. Venetia, Gio. Battista Ciotti, 1617. 5 acts; verse.

Le pompe funebri, ouero Aminta, e Clori: fauola silvestre. Ferrara, Vittorio Baldini, 1599. 5 acts; verse. 4 intermedii.

Cresci, Pietro

Tullia feroce: tragedia. Venetia, Gio. Battista Sommasco, 1591. 5 acts; verse.

Datтomo, Antonio

La maga con Fritellino mago a caso: comedia. Venetia, Domenico Louisa, [n.d.]. 3 acts; prose.

Decio, Antonio

Acripanda: tragedia. Venetia, Paolo Ugolino, 1592. 5 acts; verse.

- Firenze, Stamperia del Sermartelli, 1592. 
Il Desioso della Congrega degl' Insipidi di Siena (Domenico Tregiano)

Il consiglio villanesco, mascherata sopra tutte l'arti. Siena, 1583. Verse.

La Fortunia: comedia. Siena, Loggia del Papa, 1583. 3 acts; verse.

Il giusto inganno: comedia. Siena, Loggia del Papa, 1583. 3 acts; verse.

Gl' inganni villaneschi: egloga rusticale. Recitata per Siena il di 6. di Maggio, 1576. [n.p., n.d.]. Verse.

Gl' intrighi amorosi: commedia villesca. Siena, Loggia del Papa, 1587. 3 acts; prose.

Il ladro Cacco: favola pastorale. Venetia, Gio. Battista Ciiotti, 1583. 3 acts; verse. 3 intermedii.

Liberatione d'Amore: commedia pastorale di maggio. Siena, [n.d.]. 3 acts; verse.

Senafila: comedia pastorale. [Siena, 1576]. 3 acts; verse. Tita: egloga rusticale. Siena, 1583. 3 acts; verse.

Divitio, Bernardo da Bibbiena

Calandra: comedia. [Vinegia, Nicolo d'Aristotile detto Zoppino], 1530. 5 acts; prose.

- [Vinegia, Francesco Bindoni e Mapheo Pasini], 1547.

- [Venetia, Plinio Pietrasanta, 1554]. No t.p. In Girolamo Ruscelli's Delle comedie elette (1554).

- Fiorenza, [ i Giunti], 1558. Colophon: 1559.

—. Venetia, gli heredi di Bortolamio Rubin, 1586.

- Venetia, Lucio Spineda, 1600. 
Dolce, Agostino

Almida: tragedia. Udine, Gio. Battista Natolini, 1605. 5 acts; verse.

\section{Dolce, Lodovico}

Comedie. Il ragazzo, Il marito, Il capitano, La Fabritia, Il ruffiano. Vinegia, Gabriel Giolito de' Ferrari, 1560. All 5 acts. Il capitano and Il marito in verse; others prose. Separate t.p.'s for all except Il ragazzo.

Le tragedie. Giocasta, Medea, Didone, Ifigenia, Thieste, Hecuba. Venetia, Domenico Farri, 1566. All 5 acts; verse. Separate t.p.'s for all except Giocasta.

Il capitano: comedia. Con la favola d'Adone. Vinegia, Gabriel Giolito de Ferrari, 1547.

—. Venetia, Gio. Battista Combi, 1620.

Didone: tragedia. Vinegia, Aldus, 1547. Colophon: In casa de' figliuoli di Aldo.

- Venetia, Domenico Farri, 1566. Same text as in Le tragedie.

Giocasta: tragedia. Vinegia, Aldi filii, 1549.

La Hecuba: tragedia tratta da Euripide. Venetia, Gabriel Gioli di Ferrarij, 1543. Same play as Hecuba in Le tragedie.

Ifigenia: tragedia. Vinegia, Gabriel Giolito de Ferrari e Fratelli, 1551.

—. Venetia, Gio. Battista, e Gio. Bernardo Sessa, $\chi$ 1597.

Il marito: comedia. Vinegia, Gabriel Giolito de Farrari, $\times 83$ 1547. 
Il marito: comedia. Venetia, gli heredi di Bortolamio Rubin, 1586.

La Medea: tragedia. Vinegia, Gabriel Giolito de' Ferrari, 1558.

. Vinegia, Gabriel Giolito de' Ferrari, 1560.

鲌. Venetia, Domenico Farri, 1566. Same text as in Le tragedie. This is not the same play as Medea in Le tragedie di Seneca tradotte da M. Lodovico Dolce, Venetia, Gio. Battista et Marchion Sessa F., [1560].

Thyeste: tragedia tratta da Seneca. Venetia, Gabriel Gioli di Ferrarij, 1543. This is not the same play as Thieste in Le tragedie di Seneca tradotte da M. Lodovico Dolce.

\section{Domenichi, LoDovico}

Le due cortigiane: comedia. Venetia, Domenico Farri, 1567. 5 acts; prose.

Progne: tragedia. Fiorenza, i Giunti, 1561. No division into acts; verse. A translation of the fifteenth-century Latin play by Gregorio Corraro.

Dovizi, Bernardo

See Divitio

Epicuro, Marc Antonio, Napolitano

Cecaria: tragicomedia. Aggiontoui un bellissimo lamento del Geloso con la Luminaria. [Vinegia, Nicolo d'Aristotile detto Zoppino], 1535. Verse.

$\times 833$ EP4 40153. [Vinegia, Vettor Rauani e Compagni, 1538].

-. Venetia, gli heredi di Bortolamio Rubin, 1586.

—. Venetia, Gio. Battista Bonfadino, 1594. 
Dialogo di tre ciechi. [Vinegia, Giouanni Antonio e fratelli da Sabbio, 1526]. Verse.

Falotico della Congrega de' Rozzi (Giovanni Battista Sarto)

Il bruscello et Il boschetto. Siena, Luca Bonetti, 1574. Verse. Two rustic comedies.

\section{Falteri, Orazio}

Trionfo di Cristo, nella domenica delle palme: opera spirituale, e deuota. Siena, Loggia del Papa, 1609. 3 acts; verse. 3 intermedii.

Faustini, Giovanni

La Calisto: drama per musica. Venetia, il Giuliani, 1651. 3 acts; verse.

Felisi, Felice Agostino

Rappresentationi sacre delli quindeci misterij del Santiss. Rosario. Torino, Gio. Guglielmo Tisma, e Gio. Battista Zauatta, 1637. Verse.

Fenarolo, Ludovico

Il Sergio: comedia. Venetia, Bolognino Zaltieri, 1562. 5 acts; prose.

Venetia, Francesco Ziletti, 1584.

Ferrari, Benedetto

Poesie drammatiche. L'Andromeda, La maga fulminata, L'Armida, Il pastor regio, La ninfa avara, Il prencipe 
giardiniero, Prosperpina rapita (Intermedio). Drammi musicali. Milano, Gio. Pietro Ramellati, 1644. All except the intermedio in 3 acts; verse.

Fiamma, Paolino

Christo morto: tragedia. Venetia, Gio. Antonio Giuliani, 1644. 5 acts; verse.

Filippo, Publio, Mantovano

Formicone: comedia. [n.p., n.d.]. 5 acts; prose.

—. [Vinegia, Marchio Sessa, 1534].

Firenzuola, Agnolo

Comedie. La trinutia, I Lucidi. Separate t.p. and separate pagination for I Lucidi, 1560. Vinegia, Gabriel Giolito de' Ferrari, 1561. Both 5 acts; prose.

I Lucidi: comedia. Firenze, [i Giunti], 1552.

$x 85=$. Venetia, Bartholomeo Carampello, 1597.

- Colophon: Firenze, i Giunti, 1552. Reprinted in Raccolta di commed. toscane, [1720?].

La trinutia: comedia. Fiorenza, [Bernardo Giunti], 1549.

- Fiorenza, [li heredi di Bernardo Gionti], 1551.

- [n.p., n.d.]. Reprinted in Raccolta di commed. toscane, [1720?].

Fonsi, Francesco

$x 85377350$ Despecti damore: tragedia. [Siena, Michelagnolo di Bar[tolommeo] F[iorentino]. Et ad instantia di Giouanni dAlexandro. 1520]. 5 acts; verse. 
ForNARIS, FABRITIO DE

Angelica: comedia. Parigi, Abel l'Angelier, 1585. 5 acts; prose.

Franceschi, Pietro Antonio

La gelosia: commedia. [Siena, Simione di Niccolo, 1518]. Verse.

- [Siena, Francesco di Simeone, e Compagni. Ad istantia di Giouanni d'Alisandro. 1549].

Fuligni, Valerio

Bragadino: tragedia. Pesaro, Girolamo Concordia, 1589. 5 acts; verse.

Il Fumoso della Congrega de' Rozzi (Silvestro Cartaio) Batecchio: comedia di maggio. Siena, [n.d.]. 4 acts; verse. Capotondo: commedia nuoua rusticale. Siena, [Francesco di Simeone, ad istantia di Giouanni libraro], 1550. 3 acts; verse.

—. Siena, Loggia del Papa, [n.d.].

Panecchio: commedia nuoua di maggio. Siena, [n.d.]. Verse.

Il trauaglio: comedia. Siena, Loggia del Papa, 1580. 5 acts; verse.

Gabiani, Vincenzo

I gelosi: comedia. Vinegia, Gabriel Giolito de Ferrari e Fratelli, 1551. 5 acts; prose. 
Gabrieli, Angelo (Armentoldo Sampognano)

I Gli amorosi sospetti: fauola pastorale. Venetia, Gio. Battista Ciotti, 1605. 5 acts; verse.

Ciro monarca di Persia: tragedia. [Venetia, gli Heredi di Pietro Farri, 1628]. 5 acts; verse.

\section{Gabrielli, Gabriello}

L'innocente fanciulla: comedia. Venetia, Ghirardo Imberti, 1629. 5 acts; prose.

Galanni, Gioseppo Leggiadro, da Parma

La Portia: comedia. [n.p., n.d.]. 5 acts; prose.

Galladei, Maffeo

Medea: tragedia. Venetia, Giouan. Griffio, 1558. 5 acts; verse.

Gallo, Phylenio

Eglogha pastorica asdrucciolo di Phylenio Gallo. [Siena, $\mathbf{M}$ [ichelagnolo] di B[artolommeo] $\mathbf{F}$ [iorentino]. Ad instantia di M. G[iovanni] di A[lesandro], 1524]. Verse.

\section{Gattici, Francesco}

Le pazzie giovenili: comedia. Venetia, Gio. Battista Combi, 1624. 5 acts; prose.

Gelli, Grovanni Battista

Lo errore: [commedia]. [n.p., n.d.]. Reprinted in Raccolta di commed. toscane, [1720?]. 
/ La sporta: comedia. Fiorenza, i Giunti, 1566. 5 acts; prose.

. Colophon: Firenze, i Giunti, 1602. Reprinted in Raccolta di commed. toscane, [1720?].

Polifila: comedia. See under Anonymous Plays.

Ghirardelli, Grovanni Battista Filippo

3 Il Costantino: tragedia. Con la Difesa della medesima. Seconda editione. Roma, a spese di Gregorio, e Giouanni Andreoli, 1660. 5 acts; prose.

GHIRARDI, BoNETo

५ La Leonida: comedia. Venetia, Paolo Meietto, 1585. 5 acts; prose.

\section{Ghirlandi, Fulvio}

5 Gli amorosi travagli: comedia. Fiorenza, Cosimo Giunti, 1600. 5 acts; prose.

\section{Giancarli, Gigio Arthemio}

6 La capraria: comedia. Venetia, Francesco Marcolini, 1544. 5 acts; prose.

\section{7 —. Venetia, [Bartolomeo Cesano], 1553.}

8 La cingana: comedia. Vinegia, Agostino Bindoni, 1550. 5 acts; prose.

\section{Giraldi Cinthio, Giovanni Battista}

9 Le tragedie. Orbecche, Altile, Didone, Antivalomeni, Cleopatra, Arrenopia, Euphimia, Epitia, Selene. Venetia, Giulio Cesare Cagnacini, 1583. Colophons of Orbecche, Eu- 
phimia, Selene: Paulo Zanfretti, 1583. Colophons of Gli Antivalomeni, Epitia: Nicolo Moretti, 1583. All 5 acts; verse. Separate t.p.'s and separate pagination for each.

I Arrenopia: tragedia. Venetia, Giulio Cesare Cagnacini, 1583. Same text as in Tragedie.

Cleopatra: tragedia. Venetia, Giulio Cesare Cagnacini, 1583. Same text as in Tragedie.

Egle: satira. [n.p., n.d.]. 5 acts; verse.

Epitia: tragedia. Venetia, Guilio Cesare Cagnacini, 1583. Same text as in Tragedie.

Orbecche: tragedia. [Vinegia], Aldus, 1543. Colophon: In casa de figliuoli d'Aldo, Vinegia, 1543.

[_. [n.p., 1547].

. Vinegia, Gabriel Giolito de Ferrari e Fratelli, 1551. Colophon: 1552.

—. Venetia, Francesco Rampazetto, [1564].

\section{Giusti, Vicenzo}

Elpina: favola pastorale. Udine, [Gio. Battista Natolini], 1595. 5 acts; verse.

Fortunio: comedia. Venetia, Nicold Moretti, 1593. 5 acts; prose.

\section{Glissenti, Fabio}

Sarcodinamia, ciò̀ La possanza della carne: favola morale. Venetia, Marco Ginami, 1620. 5 acts; verse.

\section{Gnavio, Caio}

Generositd d'amore: comedia. Venetia, Ghirardo Imberti, 1629. 5 acts; prose. 
Goselini, Gruliano

Amore della Patria: componimento scenico. Venetia, Barezzo Barezzi, 1604. 5 acts; verse. Except for the prologue, this is an almost verbatim reprinting of Aretino's Orazia.

Grassi, Simone

Il Roggiero sovrano della Calabria: opera tragicomicamorale. Bologna, il Longhi, 1693. 3 acts; prose.

Grasso, Nicola

Eutychia: comedia. [Roma, 1524]. 5 acts; prose.

Gratarolo, Bongianni

Altea: tragedia. Vinegia, Francesco Marcolini, 1556. 5 acts; verse.

Gratiani, Girolamo, conte

Il Cromuele: tragedia. Bologna, li Manolessi, 1671. 5 acts; verse.

Grazzini, Antonio Francesco (Il Lasca)

L'Arzigogolo: commedia. 5 acts; prose. In Teatro comico fiorentino (1750). First printing.

La gelosia: comedia. Firenze, [i Giunti], 1551. 5 acts; prose.

- Venetia, Bernardo Giunti, e Fratelli, 1582.

I parentadi: comedia. Venetia, Bernardo Giunti, e Fratelli, 1582. 5 acts; prose.

La Pinzochera: comedia. Vinegia, Bernardo Giunti, e Fratelli, 1582. 5 acts; prose. 
La Sibilla: comedia. Vinegia, Bernardo Giunti, e Fratelli, 1582. 5 acts; prose.

La spiritata: comedia. Venetia, Bernardo Giunti, e Fratelli, 1582. 5 acts; prose.

La strega: comedia. Venetia, Bernardo Giunti, e Fratelli, 1582. 5 acts; prose.

\section{Groto, Luigi}

La Alteria: comedia. Venetia, Fabio, et Agostino Zoppini Fratelli, 1587. 5 acts; verse.

La Calisto: nova favola pastorale. Vinegia, Fabio, et Agostin Zoppini Fratelli, 1586. 5 acts; verse.

La Dalida: tragedia. Venetia, 1572. 5 acts; verse.

La Emilia: comedia. Venetia, Francesco Ziletti, 1579. 5 acts; verse.

- Venetia, Fabio, et Agostin Zoppini Fratelli, 1586.

La Hadriana: tragedia. Venetia, Fabio, et Agostin Zopini Fratelli, 1583. 5 acts; verse.

$\times 8536910+1599$. Venetia, Agostin Zopini, e Nepoti, 1599.

Lo Isach: rappresentation noua. Venetia, Fabio, et Agostin Zoppini Fratelli, 1586. 5 acts; verse.

Il pentimento amoroso: nuova favola pastorale. Venetia, Fabio, et Agostin Zoppini Fratelli, 1592. 5 acts; verse.

$\times 853$ G910 T Il thesoro: comedia. Venetia, Fabio, et Agostin Zopini Fratelli, 1583. 5 acts; verse.

—. Venetia, Fabio, et Agostin Zopini Fratelli, 1586.

Guarini, Alfonso di Battista

Sponsalitio: comedia. [n.p., n.d.]. 5 acts; verse. 
Guarini, Grovanni Battista

La idropica: commedia. Venetia, Gio. Batt. Ciotti, 1613. 5 acts; prose.

- [n.p., n.d.]. Reprinted in Raccolta di commed. toscane, [1720?].

Il pastor fido: tragicomedia pastorale. Venetia, Gio. Bat- $\times 8536930 \mathrm{P}$ tista Bonfadino, 1590. 5 acts; verse.

- Venetia, Gio. Battista Ciotti, 1602. This edition has the author's annotationi.

- Venetia, Gio. Battista Ciotti, 1605. A reprint of the 1602 edition.

—_. Venetia, Giouan Battista Ciotti, 1621.

- Parigi, Claudio Cramoisy, 1650.

Le berger fidelle. Traduit de l'italien de Gaurini en vers françois. Cologne, Pierre Marteau, 1671. Fictitious imprint. Italian and French texts.

1671 edition.

Cologne, Pierre Marteau, 1586. A reprint of the $\times 853$

-. Londra, Giovanni Pickard, 1718.

—. Londra, Tomaso Wood, 1728.

Guazzo, Marco

Discordia damore: tragedia. [Venetia, Francesco Bindoni, $\underset{653}{69321001526}$ e Mapheo pasyni compagni], 1526. 5 acts; verse.

Cop. 2.

- [Vineggia, Nicolo d'Aristotile detto Zoppino], $\times 853$

1528. 
Errori damore: comedia. [Venetia, Francesco Bindoni, e Mapheo Pasyini compagni], 1526. 5 acts; verse.

Miracolo d'amore: satira. [Vinegia, Nicolo d'Aristotile detto Zoppino], 1530. 5 acts; verse.

Guerrini, Francesco

La legge d'amore: comedia. Ronciglione, 1641. 5 acts; prose.

Guicciardi, GIOvanNi Maria

Il sogno: favola boschereccia. Ferrara, Vittorio Baldini, 1601. 5 acts; verse.

Guidani, Nicolo(?)

Eustachia: comedia. Vinegia, Aldo, 1570. 5 acts; prose. Dedication by Nicolo Guidani, who speaks of the author as "mio fratello." Toppi (p. 334): "publicata da un'altro Nicolò suo fratello cugino."

Guidi, Alessandro

See Cleoneo

Guidoccio, Giacomo

See Guidozzo

Guidozzo, Giacomo

Il capriccio: favola boscareccia. Venetia, Giacomo Vincenti, 1610. 5 acts; verse.

La Mathilda: tragedia. Trevisi, Domenico Amici, 1592. 5 acts; verse. 


\section{Hondedei, Giovanni}

Asmondo: tragedia. Venetia, Angelo Salvadori, 1633. 5 acts; verse.

\section{L'INFIAMMato}

See Gratiana under Anonymous Plays

\section{INGEgNeri, ANGELO}

Danza di Venere: pastorale. Vicenza, nella Stamperia Noua, 1584. 5 acts; verse.

\section{Lanci, CoRnelio}

Mestola: comedia. Fiorenza, Giorgio Marescotti, 1583. 5 acts; prose.

La Niccolosa: commedia. Firenze, Bartolommeo Sermartelli, 1591. 5 acts; prose.

Olivetta: commedia. Firenze, Stamperia del Sermartelli, $\times 853 \angle 2200$ 1587. 5 acts; prose.

Rappresentazione di S. Bastiano tratta dalla sua vita. Fi- $\times 853 L 22 \theta R$ renze, Stamperia del Sermartelli, 1585. 5 acts; prose.

\section{Landi, Antonio}

[Il commodo: comedia]. No t.p. Apparato et feste nelle noze dello illustrissimo Signor Duca di Firenze, e della Duchessa sua Consorte, con le sue Stanze, Madriali, Comedia, et Intermedij, in quelle recitati. [Fiorenza, Benedetto Giunta, 1539]. 5 acts; prose. 5 intermedii.

—. Firenze, i Giunti, 1566. This second edition also has the intermedii. 


\section{IL LASCA}

See Grazzini

[Lauro, Ghristoforo]

Frutti d'amore: favola pastorale. Venetia, Sebastiano Combi, 1608. 5 acts; verse.

Legacai, Pietro Antonio (Lo Stricca)

Bernino: aegloga rusticale. [Venetia, Francesco Garone, 1527]. Verse.

Cicro: egloga pastorale. [Siena, Presso a san Viglio, a instantia di Giouanni di Alisandro, 1546]. Verse.

Sauina: egloga a la Martorella. [Vinegia, Girolamo Pencio, ad instantia di Christoforo da Millano ditto Stampone, 1528]. Verse.

Sauina: egloga rusticale. [Siena, Antonio Mazozochi, ad instantia di maestro Giouanni d'alexandro], 1545.

Solfinello: egloga rusticale. [Roma, Valerio Dorico], [n.d.]. Verse.

- [n.p., n.d.]. The ending of this version, called a commedia, is different from that of the above.

Leggiadro, Giuseppe Galanni da Parma

See Galanni

\section{Leonelli, Malatesta}

La Deianira: opera recitatiua in Musica. Venetia, Angelo Saluadori, 1635. 5 acts; verse. 
Leoni, Giovanni Battista

See Settizonio

Licco, Gasparo

La trionfatrice Christina: [rappresentazione sacra]. Serraualle di Vinetia, Marco Claseri, 1605. 5 acts; verse. 5 intermedii.

Lionardo detto Mescolino (Allacci calls him Lionardo di Sier Ambrogio)

Trionfo di Pan Dio de' Pastori: opera rusticale. [Siena, a S. Viglio, a instantia di Giouanni di Alisandro, 1546]. Verse. With Campani's Strascino.

\section{Liviera, Grovanni Battista}

Giustina vergine, e martire santissima: hierotragedia. Serraualle di Vinetia, Marco Claseri, 1605. 5 acts; verse.

LOMBARDI, BERNARDINO

L'alchimista: comedia. Venetia, Lucio Spineda, 1602. 5 acts; prose.

Longo, Pietro

L'allieua: comedia. Napoli, Costantino Vitale, 1618. 5 acts; prose.

Loredano, Grovanni Francesco

Lo incendio: comedia. Vinegia, alla Libraria della Speranza, 1597. 5 acts; prose. 
La malandrina: comedia. Venetia, all' insegna della Speranza, 1587. 5 acts; prose.

La matrigna: comedia. Venetia, alla Libraria della Speranza, 1601. 5 acts; prose.

La turca: comedia. Vinegia, alla Libraria della Speranza, 1597. 5 acts; prose.

Lottini, Giovanni Agnolo

Il dannoso piacere: rappresentazion morale. Firenze, Giorgio Marescotti, 1602. Colophon: Gli heredi di Giorgio Marescotti, 1602. 5 acts; verse.

Santa Agnesa: sacra rappresentazione. Firenze, Michelagnolo di Bart. Sermartelli, 1592. 5 acts; verse.

San Lorenzo: sacra rappresentazione. Firenze, Michelagnolo di Bart. Sermartelli, 1592. 5 acts; verse.

Sette beati fondatori della religione de' Servi: sacra rappresentazione. Firenze, Michelagnolo di Bart. Sermartelli, 1592. 5 acts; verse.

Lunardi, Tiberio

Il servo fidele: comedia. Vinegia, Altobello Salicato, alla Libraria della Fortezza, 1586. 3 acts; prose.

- Vinegia, Altobello Salicato, 1597.

Machiavelli, Niccolò

Clitia: comedia. 5 acts; prose. In Tutte le opere, Geneva, Pietro Aubert, 1550.

—. In Tutte le opere, [n.p.], 1550.

. Fiorenza, [i Giunti], 1556. 
- In Lasino doro, Roma, [London, John Wolfe], 1588.

La mandragola: comedia. In Tutte le opere, Geneva, Pietro Aubert, 1550. 5 acts; prose.

—. In Tutte le opere, [n.p.], 1550.

- Venetia, Plinio Pietrasanta, 1554. In Girolamo Ruscelli's Delle comedie elette (1554).

- In Lasino doro.

Maggi, Biagio

Il tradimento amoroso: comedia. Venetia, Ghirardo, e Iseppo Imberti, 1625. 5 acts; prose.

Il Malatesta

Rappresentatione spirituale del miracolo della Sacra Vergine Santa Caterina da Siena. Siena, Loggia del Papa, 1611. Verse.

Maleguzzi, Flaminio

La Theodora: comedia. Venetia, Domenico Farri, 1572. 5 acts; verse.

Malmignati, Giulio

Il Clorindo: tragedia pastorale. Treviso, Aurelio Reghettini, 1604. 5 acts; verse.

Manfredi, Mutio

Il contrasto amoroso: pastorale. Venetia, Giacomo Anton. Somascho, 1602. 5 acts; verse. 
La Semiramis: tragedia. Bergamo, Comin Ventura, 1593. 5 acts; verse.

Maniscalco (Francesco Mariano Trinci)

Comedia di Amore contra Auaritia e Pudicitia intitolata il Bichiere. [Vineggia, Giouanmaria Pinardo, 1526]. Verse.

- Siena, [n.d.].

Motti di Fortuna: comedia. [Venetia, Francesco Garone, 1527]. Verse.

Pieta d'amore: comedia. Siena, [n.d.]. Verse.

Vitio muliebre: comedia. [Venetia, Francesco garone, 1527]. Verse.

- [Siena, Michelagnolo di Bernardino Castagni, ad instantia di Giouanni di Alizandro libraio, 1532].

Manzano, Scipione di

Aci: favola marina. Venetia, Gio. Battista Ciotti, 1600. 5 acts; verse.

Manzini, Grovanni Battista

Flerida gelosa: tragedia. Venetia, Andrea Baba, 1632. 5 acts; verse.

\section{Marliani, Hercole}

Le tre constanti: comedia. Mantova, Aurelio, e Lodouico Osanna, fratelli, 1622. 5 acts; prose.

[MARTinelli, Simone]

Giosef fliuolo di Giacob: rappresentatione. Bologna, gl'Eredi del Pisarri, [n.d.]. Verse. 
Marzi, Giovanni Battista

La fanciulla: commedia. Bologna, Giovanni de' Rossi, [1574]. 5 acts; prose.

Ottauia furiosa: commedia. Fiorenza, Filippo Giunti, 1589. 5 acts; prose.

\section{Massucci, Niccolò}

La Costanza: tragedia. Co'l velettaio commedia del medesimo. Fiorenza, li Giunti, 1585. 5 acts; verse.

Il velettaio: commedia. Firenze, li Giunti, 1585. 5 acts; verse. Bound and paged with La Costanza.

- Another copy without La Costanza.

MATERIALE Intronato

See Bargagli

MEDICI, LORENZINO DEI

Aridosio: comedia. Vinegia, Matio Pagan, [n.d.]. 5 acts; prose.

Colophon: Firenze, i Giunti, 1605. Reprinted in Raccolta di commed. toscane, [1720?].

Memmolo, Decio

La Susanna: tragedia. Roma, Lodouico Grignani, 1632. 5 acts; verse.

Mercati, Francesco

Il sensale: comedia. Fiorenza, i Giunti, 1561. 5 acts; prose. 
Mescolino

See Lionardo detto Mescolino

Miani, Valeria

Amorosa speranza: fauola pastorale. Venetia, Francesco Bolzetta, 1604. 5 acts; verse.

Miari, Alessandro

Il prencipe Tigridoro: tragedia. Reggio, Hercoliano Bartoli, 1591. 5 acts; verse.

Micalori, IAcomo

X854 M580N Le nozze finte: comedia. Pesaro, Flaminio Concordia, 1618. 5 acts; prose.

Moderati, Francesco

La finta schiavetta: comedia. Venetia, Angelo Saluadori, 1626. 5 acts; prose.

Giardiniera: comedia. Venetia, Alessandro Vecchi, 1615. 5 acts; prose.

Mondella, Francesco

Isifile: tragedia. Verona, Sebastiano, e Giouanni dalle Donne, 1582. 5 acts; verse.

Monte, Conte di, Vicentino

Antigono: tragedia. Venetia, Comin da Trino di Monferrato, 1565. No division into acts; verse. 
Moro, Mauritio

Il figliuolo prodigo: rappresentatione. Venetia, Carlo Pipini, 1585. Verse.

Morone, Bonaventura

La Giustina: tragedia spirituale. Venetia, Gio. Battista Combi, 1634. 5 acts; verse. 4 "tramezi spirituale."

NARDI, IACOPO

Amicitia: comedia. [n.p., n.d.]. 5 acts; verse. Goff (Incunabula in American Libraries): "[Florence: Lorenzo Morgiani and Johannes Petri, about 1496-97.]"

Negro, Francesco, Bassanese

Libero Arbitrio: tragedia. Editione seconda. [n.p.], 1550. 5 acts; prose.

NEgro, Marin

La pace: comedia. Venetia, Francesco Rocca, 1561. 5 acts; prose.

Noci, Carlo

La Cinthia: favola boscareccia. Venetia, la Compagnia $\times 854$ N670C159 Minima, 1596. 5 acts; verse.

Notturno Napolitano

Gaudio d'amore: comedia. [Vinegia, Marchio Sessa, 1531].

-. [n.p., n.d.]. 


\section{Oddi, SFORZA DEgLI}

$\times 853 O D L O E$ 1595 $\times 85 ; 0210 M$
1582

L'erofilomachia, overo il duello d'amore, et d'amicitia: comedia. Fiorenza, Filippo Giunti, 1595. 5 acts; prose. I morti vivi: comedia. Venetia, Gio. Battista Sessa, e fratelli, 1582. Colophon: gli Heredi di Marchiò Sessa. 5 acts; prose.

$\times 8530019921592$ Prigione d'amore: commedia. Fiorenza, Filippo Giunti, 1592. 5 acts; prose.

$\times 8530010$ PRi60Z. Venetia, gl'Heredi di Domenico Farri, 1607.

\section{Olradi, Angelo degli}

Cura d'amore: comedia pastorale. [Roma, Valerio Dorico, et Luigi fratelli, 1549]. Verse.

Desiata pace: comedia pastorale. [Roma, Valerio Dorico, et Luigi fratelli, 1547]. Verse.

Ondedei, Giovanni

See Hondedei

Ongaro, Antonio

×854 ON230A Alceo: favola pescatoria. Venetia, Francesco Ziletti, 1582. 5 acts; verse.

$\times 8540 \mathrm{~N} 35 \mathrm{~A} 1887$. Ferrara, Ad instanza di Alfonso Caraffa, 1587. Colophon: Appresso Giulio Cesare Cagnacini, e Fratelli, 1587.

—. Ferrara, Vitt. Bald[ini], 1614. Con gl'Intramezzi del Sig. Caualier Batista Guarini. Descritti, e dichiatati dall' Arsiccio Accademico Ricreduto. Aggiuntici appresso alcuni Discorsi del medesimo Arsiccio sopra ciascheduno Intramezzo. Lacks prologue of 1582 and 1587. 
[Ottonaio], Grovanni Battista dell'

La ingratitudine: comedia. Fiorenza, [i Giunti], 1559. 5 acts; verse.

\section{Pacinelli, Antonio}

I contrasti d'amore, e di vendetta: opera comica. Perugia, gl'Heredi di Sebastiano Zecchini, 1673. 5 acts; prose.

\section{Pallavicino, SForza}

Ermenegildo martire: tragedia. Roma, gli Eredi del Corbelletti, 1655. 5 acts; verse. A defense of the tragedy, addressed to Agostino Favoriti, is appended.

\section{Panciatichi, Vincenzo}

L'amicizia costante: tragicomedia pastorale. Fiorenza, Fi- $\times 854$ P19 of lippo Giunti, 1600. 5 acts; verse.

Gli amorosi affanni: fauola pastorale. [Venezia?], Gio. Battista Ciotti, 1606. Dedication: Di Fiorenza li 25 di Febraio 1605. 5 acts; verse.

Il re Artemidoro: tragedia. [n.p., n.d.]. Dedication: Di Fiorenza li 25 d'Agosto 1604. 5 acts; verse.

\section{Parabosco, Girolamo}

Comedie. La notte, Il Viluppo, I contenti, L'hermafrodito, Il pellegrino, Il marinaio. Vinegia, Gabriel Giolito de' Ferrari, 1560. All 5 acts; all prose except $I l$ pellegrino. Separate t.p.'s for all except La notte; separate pagination.

La fantesca: comedia. Vinegia, Gio. Battista, e Gio. Bernardo Sessa, 1597. 5 acts; prose. 


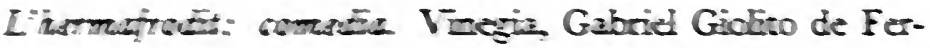
rari $15 \div 9$.

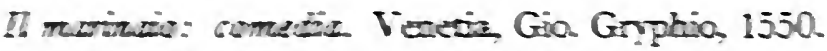

- Venesia gis heredi di Bortolamio Rubin, 1586.

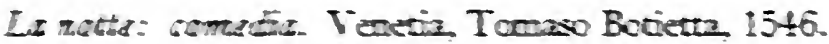

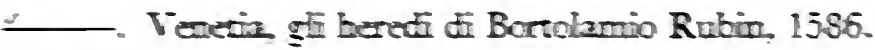

'Il gullegrizo: comzet. Vinesia, Gabriel Giolito de' Ferrati. 1500. Sume tex ax in Cometie. Last kaf belonss to Les rated.

- Vectia gis herefi di Bortalamio Rubin, 1565.

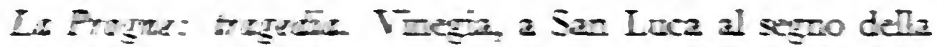

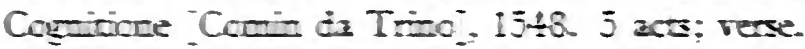

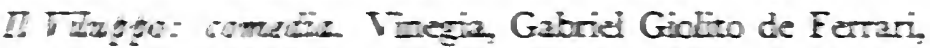
$15+2$

Pxispitico - Aluts

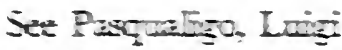

Pasperitco, Itact

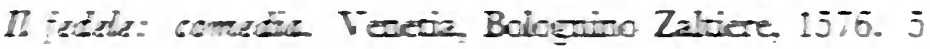
ict: prose.

- Vectio Francesco ZZleti. 15ig.

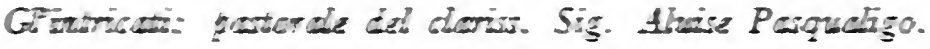
Venetis Francesco Zileti, 1581. j act: retse.

Psitor Momozonthlio (z)

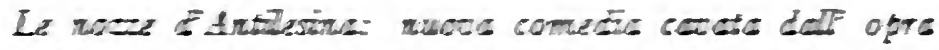

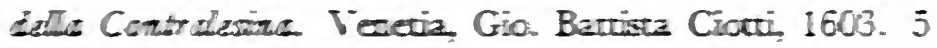


acts; prose Appended (continuover pagnaticn) to La comtrelesine, B.P.C. Sanese, Venetia, 1604.

Pisso, Horermo

Il mel merito: compdis Nizpolfy, Gia Dorm Rouca Eliobl, 1623. 5 acts; prose

Pescatcre, Gromanod Batmista

Nins: comedie Vinegia, Comin da Trion 15j2. 5 acs; verse

Pascatri, Orlarto

II Cener: tregedie. Vecon Gerclamo Discepaid 159s. I. acts; verse.

Pemirpo, Pceino

See Fintero

Procoloven, AItssenviso (Lo Stortio)

Alesserdro: comedie Vinesgia Astatio Brador 1550 5 acts; prose.

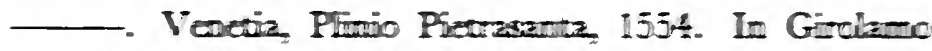

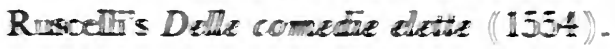

Vincgia, Gabriel Grotero de" Fenrari, 1562

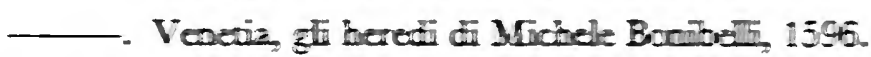

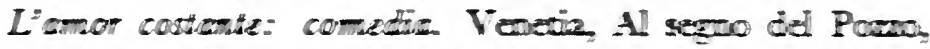
1550. Colophon: Venetia Bartrolcento Cesano, 1549. 5 acks; prose. 
| L'amor costante: comedia. Venetia, Plinio Pietrasanta, 1554. Colophon: Venetia, Per Simon, Rocca a San Polo, 1565. In Girolamo Ruscelli's Delle comedie elette (1554). L'Hortensio. See under Anonymous Plays.

\section{Pico, Girolamo}

X954 P5804 Honesta schiava: comedia. Con gli Intermedij apparenti del Sig. Gio. Francesco Pico, suo fratello. Vinegia, Altobello Salicato, 1601.5 acts; prose. 5 intermedii in verse.

\section{Pino, Bernardino}

L'Eunia: ragionamenti pastorali. Venetia, Paolo Meietti, 1582. 5 parts; prose.

$\times 8538660$ I falsi sospetti: comedia. Venetia, gli Heredi di Marchiò Sessa, 1588. Colophon: Giorgio Angelieri ad instantia di Gio. Battista Sessa, e Fratelli. 5 acts; prose.

$\times 853 \quad$ Gl'ingiusti sdegni: comedia. Vinegia, Gabriel Giolito de' Plog60 1560 Ferrari, 1560. 5 acts; prose.

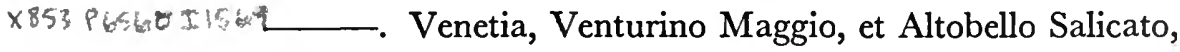
1569.

$\times 853865601626$

- Venetia, Lucio Spineda, 1607.

$\times 953$ P 651563 Lo Sbratta: comedia. Venetia, Francesco Rampazetto, 1563. 5 acts; prose.

x433 P 6560515 . Venetia, gli heredi di Bortolamio Rubin, 1586.

$\times 853$ P4.560\$160 . Venetia, Lucio Spineda, 1603.

Pistola, Antonio da (Antonio Cammelli)

[Philostrato e Pamphila]: tragedia. [Firenze?], [I stampata 
ad instantia di M. F[rancesco] Benuenuto], [n.d.]. 5 acts; verse.

Podiani, Francesco

Gli schiaui d'amore: comedia. Perugia, gli Accademici $\times 854$ Augusti, [1607]. 5 acts; prose.

- Venetia, Giouanni Alberti, 1607.

Podiani, Mario

I megliacci: [comedia]. [Peroscia, Girollamo Cartolai, 1530]. 5 acts; prose.

Poggi, Beltramo

La Cangenia: tragicomedia. Fiorenza, i Giunti, 1561. 5 acts; verse. 5 intermedii.

La inuentione della croce di Giesu Christo: [operetta tragi- $\times \$ 53$ comica]. Fiorenza, i Giunti, 1561. 5 acts; verse. 5 inter- P75 teI mediary madrigals.

Porta, Cesare della

Delfa: tragedia. Cremona, Christoforo Draconi, 1587. $5 \times 85^{3}+15100$ acts; verse.

Porta, Giovanni Battista della

Lo astrologo: comedia. Venetia, Pietro Ciera, 1606. 5 acts; prose.

Cop. 2.

La Cintia: comedia. Venetia, Gio. Battista Combi, 1628. 5 acts; prose. 
Gli duoi fratelli rivali: comedia. Venetia, Gio. Batt. Ciotti, 1601. 5 acts; prose.

La fantesca: comedia. Venetia, Gio. Battista Bonfadino, P2328F1512 1592. 5 acts; prose.

$\leftrightarrow \quad$ Venetia, Gio. Battista Bonfadino, 1596.

\section{7.}

La sorella: comedia. Venetia, Giouanni Alberti, 1607. 5 acts; prose.

$\times 854$

La trappolaria: comedia. Vinegia, Gio. Battista, e Gio. Bernardo Sessa, 1597. 5 acts; prose.

La Turca: comedia. Venetia, Pietro Ciera, 1606. 5 acts; prose.

Porta, Malatesta

I santi innocenti: tragedia. Rimino, Giouan Simbeni, 1604. 5 acts; verse.

[Pulci, Bernardo]

La rappresentatione di Barlaam et Iosofat. Firenze, alle Scalee di Badia, [n.d.]. Verse.

Quaretta, Grovanni Antonio

X854 Q260M La Margherita d'Antiochia: tragedia sacra. Parma, Mario Vigna, 1648. Engraved t.p., dated 1647, added. 5 acts; verse.

Razzi, Girolamo

La balia: comedia. Fiorenza, i Giunti, 1564. 5 acts; prose. 
La Cecca: comedia. Fiorenza, il Figliuoli di Lorenzo Tor- $\times 993$ RateC rentino, a stanza di Giorgio Marescotti, 1563. 5 acts; prose.

. Venetia, Michele Bombelli, 1596.

$\times 8532801540$

- Vinegia, Daniel Bisuccio, 1602. This edition, entitled La Zecca, has several changes in the text. Allacci calls it an edizione scorrettissima.

La Gismonda: tragedia. Fiorenza, Bartholomeo Sermar- $\times 953$ R.2106I telli, 1569. 5 acts; verse.

La Gostanza: comedia. Firenze, Cosimo Giunti, 1604. $5 \times 853$ acts; prose.

$1.210<16004$

Il Resoluto da Siena (Angiolo Cenni)

Calindera: commedia rusticale. Siena, 1546. Verse. $\quad \times 85842800$

RetTori, Assuero

Lo schiavo: comedia. Siena, Luca Bonetti, 1578. 5 acts; prose.

Rigchi, Agostino I tre tiranni: comedia. [Vinegia, Bernardino de Vitali], $\times 853,8358 \mathrm{~m}$ 1533. 5 acts; verse.

- Cop. 2.

- Cop. 3.

$\times 853230 \mathrm{~T}$ c. 2 .

X853 R.350r $c .5$

Riccio, Giovanni Giacomo

Il maritaggio delle muse: poema drammatico. Orvieto, Michel' Angelo Fei e Rinaldo Ruusi, 1625. 5 acts; verse. 4 intermedii in Italian and Latin.

La poesia maritata: comedia allegorica. Roma, Francesco Caualli, 1632. 5 acts; verse. 
Rinugcini, Ottavio

L'Arianna: tragedia. Venetia, Bernardo Giunti, Gio. Battista Ciotti, e Compagni, 1608. No division into acts; verse. L'Euridice: [tragedia]. Fiorenza, Cosimo Giunti, 1600. No division into acts; verse.

Romei, Alfonso

×954 R.64408 Gli affitti consolati: comedia. Ferrara, Vittorio Baldini, 1604. 5 acts; prose.

Roncaglia, Grovanni

Piglia il peggio: comedia. Siena, Loggia del Papa, 1580. 5 acts; verse.

Scanniccio: commedia della Speranza. Siena, Loggia del Papa, 1581. 3 acts; verse.

Roncaglia, Marcello

Pescatore: commedia rusticale. [Siena, Francesco di Simione et Compagni, ad instantia di Giouanni di Alixandro, 1547]. Verse.

Pieta d'amore: comedia [rusticale]. [n.p., n.d.]. No t.p., no colophon. Verse. Bound, but separately paged, with Giovanni Roncaglia's Piglia il peggio (1580). Allacci cites an edition of 1542 at Siena.

Roselir, Lessadro (Alessandro)

La rappresentazione di Sansone. [n.p., n.d.]. Verse.

Rugellai, Giovanni

Rosmunda: tragedia. [Venetia, Nicolo d'Aristotile detto Zoppino, 1528]. 5 acts; verse. 
- [Venetia, Nicolo d'Aristotile detto Zoppino], 1530.

- Venetia, al Segno del Pozzo, 1550. Colophon: In Venetia per Bartholomeo Cesano, 1549.

. Fiorenza, i Giunti, 1568.

. Cop. 2.

Ruschi, Flamminio

I successi d'amore: commedia. Fiorenza, Stamperia de Landini, 1633. 5 acts; prose.

\section{Ruzzante (Angelo Beolco)}

Tutte le opere. Rhodiana [by Andrea Calmo], Anconitana, Piovana, Vaccaria, Moschetta, Fiorina, Due dialoghi in lingua rustica, Dialogo facetissimo et ridiculosiss[i]mo. 1584. Dedication: "Vicenza, Giorgio Greco Stampatore." All except the dialogues in 5 acts; all prose. All have separate t.p.'s.

Anconitana: comedia. Vinegia, Stephano di Alessi, 1555. -. Vicenza, Domenico Amedio, 1617.

Due dialoghi in lingua rusticale. Vinegia, Stefano de Alessi, 1551. Colophon: In Vinegia per Bartholomeo Cesano, 1551. The first dialogue is the Parlamento.

-. Vincenza, Domenico Amadio, 1617.

Fiorina: comedia. Vinegia, Stefano di Alessi, 1556. $\times 953$ B4510 1556 -. Vicenza, Domenico Amadio, 1617.

Moschetta: comedia. Venetia, Stephano de Alessi, 1555. $\times 8538493401555$ -. Vicenza, gli Heredi di Perin, 1598.

$\times 85.384510 \mathrm{M} 1598$

Piovana: comedia, overo noella del Tasco. Vinegia, Ga- $\times 853$ briel Giolito de Ferrari et Fratelli, 1552. 
Piovana: comedia, overo noella del Tasco. Vicenza, Domenico Amadio, 1617.

Rhodiana: comedia. See under Calmo

$\times 853$ B4510v1555 Vaccaria: comedia. Vinegia, Stephano di Alessij, 1555.

X85384560 . Vinegia, Domenico de Farri, 1561.

$\times 853$.

Sacco, Tibortio, Dossetano

Sosanna: tragedia nova, raccolta da Daniello Profeta. [Bressa, Damiano di Turlini, 1537]. 5 acts; verse.

Sacrati, Scipione, Marchese

La Ciméné: drama per musica. Manuscript in seventeenthcentury hand. 5 acts; verse.

SAlvadori, ANDrea

Il Medoro rappresentato in musica nel palazzo del Serenissimo G. Duca di Toscana in Fiorenza. Fiorenza, Pietro Cecconcelli, 1623. 3 acts; verse.

Salviati, Lionardo

Due commedie. Il Granchio, e La Spina. Firenze, Stamperia di Cosimo Giunti, 1606.

Il Granchio: commedia. Firenze, [i figliuoli di Lorenzo Torrentino, e Carlo Pettinari compagno], 1566. 5 acts; verse. With intermedii.

La Spina: comedia. Ferrara, Benedetto Mammarelli, 1592. 5 acts; prose.

Sampognano, Armentoldo

See Gabrieli 
Sarto, Giovanni Battista

See Falotico

Savaro, Giovanni Francesco

L'Emiddio: tragedia. Bol[ogna], il Monti, 1666. 5 acts; verse.

Scardova, Pietro Martire

Due comedie. La naue: fauola maritima. Il cornacchione: comedia pastorale. Bologna, Anselmo Giaccarelli, 1554. Both 5 acts; prose. Separate t.p. for Il cornacchione.

Scarpella Bergamasco

See Calmo

SEGCHI, Niccolò

La camariera: comedia. Venetia, Cornelio Arriuabene, 1583. 5 acts; prose.

Glinganni: comedia. Fiorenza, i Giunti, 1562. Colophon: In Fiorenza appresso gli heredi di Bernardo Giunti, 1568. 5 acts; prose.

L'interesse: comedia. Venetia, Francesco Ziletti, 1581. 5 acts; prose.

Segni, Antonio di Carlo

Eustachio Romano: tragiedia. [Impressum Florentie, Bernardo Zuchetta, 1511]. 5 acts; verse.

Selva, Lorenzo

Il libro: comedia. Padova, Lorenzo Pasquati, 1567. 5 acts; prose. 
Seragone, Lodovico

Il martirio di Santa Giuliana da Nicomedia: [tragedia sacra]. Perugia, Vincentio Colombara, Erede d'Andrea Bresciano, 1596. 5 parts; verse. Part of imprint on t.p. trimmed. Imprint also on colophon.

Settizonio, Lauro (Giovanni Battista Leoni)

Florinda: grottesca dramatica ouero favola eteroclita. Venetia, Francesco Ciotti, 1607. 5 acts; prose.

Roselmina: favola tragisatiricomica. Venetia, Pietro Miloco, 1623. 5 acts; prose.

Sicinto, Ghristoforo

La pazzia: comedia. Venetia, Gio. Battista Pulciani, 1604. 5 acts; prose.

Silvestro Cartaio

See Il Fumoso

Sinibaldi, GrovanNi

Altea: comedia. Vinegia, gli Heredi di Marchiò Sessa, 1588. 5 acts; prose.

Sogliani, Giovanni Battista

L'Uccellatoio: commedia. Coll' annotazioni del medesimo. Venetia, Giouanni Querigli, 1627. 5 acts; prose.

Sorboli, Girolamo

Celestina: favola pastorale. Ferrara, Vittorio Baldini, 1586. 5 acts; verse. 
Speroni, Sperone

Canace: tragedia. [Fiorenza, Francesco doni], 1546. No division into acts; verse.

- Vinegia, Vincenzo Valgrisi, 1546. On the title page is the following comment: "Se nel fine di questa sana, intiera, e corretta si guardera, si trouera annotato quanto lacera, tronca, e corrotta sia quella, che da altri, che da noi, e contra il uoler dell' Auttore, e senza licenza ueruna, occultamente è stata stampata."

- With Giuditio sopra la Tragedia di Canace $e$ Macareo, [Lucca, Vincentio Busdrago, 1550].

- Venetia, 1566. The Giuditio added.

- Alla quale sono aggiunte alcune altre sue compositioni, et una Apologia, et alcune Lettioni in difesa della Tragedia. Venetia, Giouanni Alberti, 1597. 5 acts; verse.

Spezzani, Antonio

Rappresentatione di S. Cecelia. Bologna, Stamperia di Gio. Rossi, 1581. 5 acts; verse. 5 intermedii.

\section{Spinello, Alessandro}

Cleopatra: tragedia. Vinegia, [Pietro de Nicolini da Sabbio], 1550. 5 acts; verse.

\section{Stanchi, Mighele}

Personaggi finti: opera scenica. Bologna, Gioseffo Longhi, 1679. 5 acts; prose.

La Rosaura: opera scenica. Macerata, li Grisei, e Gioseppe Piccini, 1666. 5 acts; prose. 
Lo Stechito da Siena (Antonio Maria Cartaio)

Farfalla: comedia. [Roma, Valerio Dorico e Louigi fretelli, 1549]. Verse.

Lo Stordito

See Piccolomini

Lo Strafalgione (Ascanio Cacciaconti)

Bel Corpo: comedia. [Siena, Ant. Mazochi ad istantia Maestro Giouanni d'Alexandro, 1544]. Verse.

Calzagallina: commedia nuova rusticale et ridicolosa. Siena, 1550. Verse.

Filastoppa: capriccio. [Siena, Antonio Mazochi ad instantia di Giouanni d'Alexandro, 1545]. Verse.

Pellagrilli: commedia. Siena, Loggia del Papa, 1630. 5 acts; verse.

Stramboli, Francesco

Il finto paggio, overo amare, e non sapere chi: operetta. Bologna, il Longhi, [n.d.]. 3 acts; prose. Allacci calls this play a tragicommedia and dates it 1686 .

Lo Strascino

See Campani

Lo Stricca

See Legacci

TANi, Nicolò

La cognata: comedia. Padoa, Paulo Meieto, 1583. 5 acts; prose. 
Tarentino, Secondo

Il capitan Bizzarro: comedia. Vinegia, Andrea Rauenoldo, 1567. 5 acts; verse.

Tasso, Torguato

Aminta: favola boscareccia. In Parte prima of Delle rime, Vinegia, [Device of Aldo], 1582. 5 acts; verse. No t.p.

- Vinetia, Aldo, 1583.

- In Parte prima of Delle rime, et prose, Vinetia, Aldo, 1583. Half t.p.; separate pagination.

-. Vinetia, Aldo, 1589.

- Ferrara, 1589. In Parte prima of Rime, et prose, Ferrara, [Vittorio Baldini] ad instantia di Giulio Vasalini, 1589. Separate t.p.; separate pagination.

Intrichi d'amore: comedia. Venetia, Gio. Battista Ciotti, 1613. 5 acts; prose. 5 intermedii by Giovanni Antonio Liberati added. Il re Torrismondo: tragedia. Bergamo, Comino Ventura, e Compagni, 1587. 5 acts; verse.

- Verona, Girolamo Discepolo, ad instantia di Marc' Antonio Palazzolo, 1587.

Tauro, Rafaele

Il fingere per vivere: opera. Napoli, Nouello de Bonis, 1673. 5 acts; prose.

L'Isabella, overo la donna più costante: comedia. Napoli, Nouello de Bonis, 1679. 3 acts; prose.

Terzo, Giovanni Battista

La Androphysia racconsolata: rappresentatione misteriosa. Bergamo, Comin Ventura, 1604. 5 acts; prose. 
Testi, Fulvio, conte

L'Arsinda overo la discendenza de' Serenissimi Principi d'Este: dramma tragicomico. Unfinished: 3 acts and part of Act 4; verse. No t.p. In Poesie liriche, Venetia, li Prodotti, 1683.

2 Componimento drammatico fatto per la musica nel giorno natalizio della Serenissima Maria Farnese duchessa di Modana. 3 acts; verse. No t.p. In Poesie liriche, Venetia, li Prodotti, 1683.

3 L'isola d'Alcina: tragedia. 5 acts; verse. No t.p. In Opere, Venetia, Giunti e Baba, 1644.

-. In Poesie liriche, Venetia, li Prodotti, 1683.

Tirinto, Accademico Rinato

La pazzia politica di Roberto Rè di Sicilia: opera scenica. Venetia, Domenico Louisa, [n.d.]. 3 acts; prose.

\section{Torelli, Giulio Cesare}

L'Anchora: comedia. Venetia, Giouanni Alberti, 1611. 5 acts; prose.

Torelli, Pomponio, conte

La Galatea: [tragedia pastorale]. Parma, Erasmo Viotti, 1603. 5 acts; verse.

$\times 85476$ on La Merope: tragedia. Parma, Erasmo Viotto, 1589. No division into acts; verse.

Il Tancredi: tragedia. Parma, Erasmo Viothi, 1605. No division into acts; verse. 
Tortoletti, Bartolomeo

Agrippina la maggiore: tragedia. 5 acts; verse.

L'Amazoni: favola regia. 3 acts; verse.

Intramezzi d'Erminia, tratti dalla Gerusalemme del Signor Torquato Tasso. 5 intramezzi; verse.

All of the above in La scena reale, Roma, Lodouico Grignani, 1645.

Tregiano, Domenico

See Il Desioso

Trinci, Francesco Mariano

See Maniscalco

Trissino, Giovanni Giorgio

La Sophonisba: tragedia. No division into acts; verse. No t.p. In Rime, [Vicenza, Tolomeo Ianiculo, 1529].

—. In Li ritratti, Vineggia, Augustino Bindoni, 1549.

- Vinegia, Gabriel Giolito de Ferrari e Fratelli, 1553.

- Vinegia, Francesco Lorenzini da Turino, 1560.

-. Vicenza, ad instantia di Girolamo Brescia, 1585.

Colophon: Appresso Perin Libraro, e Giorgio Greco compagni, 1585.

Turco, Carlo

Calestri: tragedia. Vinetia, [Device of Aldo], 1585. 5 acts; verse. 


\section{Ulivi, Pietro}

Il Bifolco: comedia villanesca. [n.p.], 1549. 5 acts; verse.

Valacca da Sarope (T. M. Cavalca)

Lo stafile per gl'abusi d'hoggidi: capriccio satiricomico. Venetia, Gio. Battista Surian, 1643. 5 acts; prose.

Valerini, Adriano

Afrodite: tragedia. Verona, Sebastiano, e Giouanni dalle Donne fratelli, 1578. 5 acts; verse.

VARChi, BENEDETTO

La suocera: commedia. 5 acts; prose. Colophon: Firenze, Bartolommeo Sermartelli, 1569. Reprinted in Raccolta di commed. toscane, [1720?].

Veniero, Maffio

Hidalba: tragedia. Venetia, Andrea Muschio, 1596. 5 acts; verse.

Venuti, Federigo

See Il Vigoroso

Veraldo, Paolo

Mascarate, et capricci dilettevoli, recitativi in comedie, et da cantarsi in ogni sorte d'instromenti operete di molto spaso. Venetia, Angelo Saluadori, 1626. Verse. 
Verlatto, Leonoro

Rodopeia: tragedia. Venetia, Francesco Ziletti, 1582. 5 acts; verse.

VerUCCI, VerGiLIO

Il dispettoso marito: comedia. Ronciglione, 1676. 5 acts; prose.

Vignali, Antonio

See Arsiccio Intronato

Il Vigoroso Accademico Unrto di Cortona (Federigo Venuti)

La vincitrice Caterina: tragedia spirituale. Manuscript. Dedication signed "Federigo Venuti." Allacci lists a printing at Firenze in 1615. 5 acts; prose.

Villifranchi, GiovanNi

Altamoro: tragedia. Fiorenza, gli Heredi di Iacopo Giunti, 1595. Colophon: 1596. 5 acts; verse.

Vinta, Francesco

La regina Ilidia: tragedia. Venetia, Gio. Battista Ciotti, 1606. 1606 stamped over 1605. 5 acts; verse.

Viviani, Viviano

L'Ortigia: tragicomedia boscareccia. Venetia, Gio. Battista Pulciano, 1607. 5 acts; verse. 


\section{Zinano, Gabriele}

L'Amerigo: tragedia. Reggio, Hercoliano Bartholi, [n.d.]. Dedication dated 1590. 5 acts; verse. The author's Discorso della tragedia (Reggio, 1590) added.

\section{Zoppio, MelGhiorre}

La Medea essule: tragedia. Bologna, gli Heredi di Giouanni Rossi, 1602. 5 acts; verse. 


\section{INDEX OF PLAYS}

Abraam et Isaac suo figliuolo, La rappresentazione di (Belcari), 11

Aci (Manzano), 44

Acripanda (Decio), 25

Adamira ouero La statua dell'honore, L' (Cicognini), 22

Adamo, $L^{\prime}$ (Andreini, Giovanni Battista), 7

Adoratione de' pastori al presepe di Christo, $L^{\prime}$ (Abati), 5

Affitti consolati, Gli (Romei), 56

Afrodite (Valerini), 66

Agia, La (Contile), 24

Aglae (Bartolommei Smeducci), 10

Agnolo Rafaello: e di Tobbia, La rappresentatione del (Anonymous), 3

Agrippina la maggiore (Tortoletti), 65

Albesinda (Campelli), 17

Alceo (Ongaro), 48

Alchimista, $L^{\prime}$ (Lombardi), 41

Allessandro (Piccolomini), 51

Allieua, L' (Longo), 41

Almida (Dolce, Agostino), 27

Altamene (Bartolommei Smeducci), 10 
Altamoro (Villifranchi), 67

Altea (Carbone), 17

Altea (Gratarolo), 35

Altea (Sinibaldi), 60

Alteria, La (Groto), 36

Altile (Giraldi Ginthio), 33

Aman, La rappresentatione di (Anonymous), 3

Amante furioso, $L^{\prime}$ (Borghini), 14

Amaranta (Casalio), 18

Amarilli, L' (Castelletti), 19

Amata (Bonifaccio, Baldassare), 13

Amazoni, L' (Tortoletti), 65

Amerigo, L' (Zinano), 68

Amicitia (Nardi), 47

Amicizia costante, L' (Panciatichi), 49

Amida tiranno (Aleardi), 5

Aminta (Tasso), 63

Amor costante, L' (Piccolomini), 51, 52

Amor venale (Bonifaccio, Gasparo), 13

Amore della Patria (Goselini), 35

Amore, opera a caso (B, M M), 10

Amore tra nemici, L' (Cicognini), 22

Amorosa speranza (Miani) , 46

Amorosi affanni, Gli (Panciatichi), 49

Amorosi inganni, Gli (Carbone), 17

Amorosi sospetti, Gli (Gabrieli, Angelo), 32

Amorosi travagli, Gli (Ghirlandi), 33

Amoroso sdegno, L’ (Bracciolini), 14

Anchora, L' ('Torelli, Giulio C.) , 64

Anconitana (Ruzzante), 57 
Andreuccio del Boccaccio ridotto al rappresentabile, $L^{\prime}$ (Canali), 17

Andromeda, $L^{\prime}$ (Ferrari) , 29

Androphysia racconsolata, $L a$ (Terzo), 63

Angelica (Fornaris), 31

Annuntiatione di nostra Donna, La rappresentazione della (Belcari), 11

Antigono (Alamanni), 5

Antigono (Monte), 46

Antivalomeni (Giraldi Ginthio), 33

Ardito vergognoso, $L^{\prime}$ (Calcolona), 15

Arianna, $L^{\prime}$ (Rinuccini), 56

Aridosio (Medici), 45

Aristippia (Anonymous), 1

Armida, L' (Ferrari) , 29

Arrenopia (Giraldi Cinthio), 33, 34

Arsinda overo la discendenza de' Serenissimi Principi d'Este, L' (Testi), 64

Arsinoe (Angeli), 7

Arzigogolo, L' (Grazzini) , 35

Asdrubale (Castellini), 19

Asmondo (Hondedei), 39

Assiuolo, L' (Gecchi), 19

Astrologo, Lo (Porta, Giovanni Battista della), 53

Avventurose disaventure, Le (Basile), 10

Balia, La (Razzi), 54

Ballo di donne turche ... (Anonymous), 1

Barlaam et Iosofat, La rappresentatione di (Pulci), 54

Barona. See Lamento pietoso 
Batecchio (Il Fumoso della Congrega de' Rozzi), 31

Beco, El (Belo), 11

Bel Corpo (Lo Strafalcione), 62

Berger fidelle, Le (Guarini, Giovanni Battista), 37

Bernardi, I (Ambra), 6

Bernino (Legacci), 40

Bichiere [Bicchiere], Il. See Comedia di Amore...

Bifolco, Il (Ulivi), 66

Boschetto, Il (Falotico della Congrega de' Rozzi), 29

Bragadino (Fuligni), 31

Bravure del Capitano Spavento, Le (Andreini, Francesco), 6

Bruscello, Il (Falotico della Congrega de' Rozzi), 29

Calandra (Divitio), 26

Calestri (Turco), 65

Calindera (II Resoluto da Siena), 55

Calisto, La (Faustini), 29

Calisto, La (Groto), 36

Calzagallina (Lo Strafalcione), 62

Camariera, La (Secchi), 59

Canace (Speroni), 61

Candelaio (Bruno), 14

Cangenia, La (Poggi), 53

Capitan Bizzarro, Il (Tarentino), 63

Capitano, Il (Dolce, Lodovico), 27

Capotondo (Il Fumoso della Congrega de' Rozzi), 31

Capraria, La (Giancarli), 33 
Capriccio, Il (Guidozzo), 38

Cassaria, La (Ariosto), 8

Catrina, $L a$ (Berni), 12

Cecaria (Epicuro), 28

Cecca, La (Razzi), 55

Celestina (Sorboli), 60

Cesare, Il (Pescetti), 51

Cesarea Gonzaga, La (Contile), 24

Chi trionfa morendo, overo $S$. Casimiro (Calcolona), 16

Chimere pitagoriche, cabaliste, chimiche . . , Le (Allè), 6

Christo morto (Fiamma), 30

Cicro (Legacci), 40

Ciméné, La (Sacrati), 58

Cingana, La (Giancarli), 33

Cinthia, La (Noci) , 47

Cintia, La (Porta, Giovanni Battista della), 53

Ciro monarca di Persia (Gabrieli, Angelo), 32

Cleopatra (Cesari), 21

Cleopatra (Giraldi Cinthio), 33, 34

Cleopatra (Spinello), 61

Clitia (Machiavelli), 42

Clodoveo trionfante (Bartolommei Smeducci), 10

Clorindo, Il (Malmignati), 43

Cofanaria, La (Ambra), 6

Cognata, La (Tani), 62

Coltellino (Campani), 16

Comedia di Amore contra Auaritia e Pudicitia intitolata il Bichiere (Maniscalco), 44 
Comedia di mandata Sophia (Brusonio), 15 Comedia intitolata sine nomine (Anonymous), 1 Comedia noua volgare di uno eccellente poeta Mantuano (Anonymous), 1

Commedia di Tozzo e Cappellina, La (Boiardo), 13

Commodo, Il (Landi), 39

Componimento drammatico ... (Testi), 64

Consiglio de gli dei, Il (Abati), 5

Consiglio villanesco, mascherata sopra tutte l'arti, Il (Il Desioso ....), 26

Contenti, I (Parabosco), 49

Contralesina, La (Pastor Monopolitano?), 50

Contrasti d'amore, e di vendetta, I (Pacinelli), 49

Contrasto amoroso, Il (Manfredi), 43

Cornacchione, Il (Scardova), 59

Corredo, Il (Cecchi), 19

Corsaro Arimante, Il (Aleardi), 5, 6

Cortegiana, La (Aretino), 7

Costantino, Il (Ghirardelli), 33

Costantino imperatore . . , La rappresentazione di (Anonymous), 3

Costanza, La (Massucci) , 45

Creso (Bartolommei Smeducci), 10

Cromuele, Il (Gratiani), 35

Cura d'amore (Olradi), 48

Dalida, La (Groto) , 36

Dannoso piacere, Il (Lottini), 42

Danza di Venere (Ingegneri), 39

Dauid sconsolato (Brunetto), 14 
Deianira, $\mathrm{La}$ (Leonelli), 40

Delfa (Porta, Cesare della), 53

Desiata pace (Olradi), 48

Desiderio, e Speranza fantastichi (Cini, Desiderio), 23

Despecti damore (Fonsi), 30

Dialogo di tre ciechi (Epicuro), 29

Dialogo facetissimo et ridiculosiss[i]mo (Ruzzante), 57

Didone (Dolce, Lodovico), 27

Didone (Giraldi Cinthio), 33

Diecimila martiri . . , La rappresentatione de (Anonymous), 3

Discordia damore (Guazzo), 37

Dispettoso marito, $\mathrm{Il}$ (Verucci), 67

Dissimili, I (Cecchi), 19

Donna costante, La (Borghini), 14

Donna più sagace fra l'altre, La (Cicognini), 22

Donzello, Il (Cecchi), 19

Dote, La (Cecchi), 19

Due cortigiane, Le (Domenichi), 28

Due dialoghi in lingua rustica (Ruzzante), 57

Duoi fratelli rivali, Gli (Porta, Giovanni Battista della), 54

Edippo (Anguillara), 7

Edipo tiranno (Angelio), 7

Egle (Giraldi Cinthio), 33

Egloga rusticale di Grechoi, et del vescouo (Anonymous), 1

Eglogha pastorica... (Gallo), 32 
Elpina (Giusti), 34

Emiddio, L' (Savaro), 59

Emilia, La (Groto), 36

Endimione, L' (Cleoneo), 23

Epitia (Giraldi Cinthio), 33, 34

Ermenegildo martire (Pallavicino), 49

Erofilomachia overo il duello d'amore, et d'amicitia, $L^{\prime}$ (Oddi) , 48

Errore, Lo (Gelli), 32

Errori, Gli (Cenci), 20

Errori damore (Guazzo), 38

Errori incogniti (Buonfanti), 15

Esaltazione della croce, con $i$ suoi intermedi, $L^{\prime}$ (Cecchi), 20

Eugenia (Bartolommei Smeducci), 10

Eunia, L' (Pino), 52

Euphimia (Giraldi Cinthio), 33

Euridice, L' (Rinuccini), 56

Eustachia (Guidani), 38

Eustachio (Bartolommei Smeducci), 10

Eustachio Romano (Segni), 59

Eutychia (Grasso), 35

Evandro, L' (Bracciolini), 14

Fabritia, La (Dolce, Lodovico), 27

Falsi sospetti, I (Pino), 52

Fanciulla, La (Marzi), 45

Fantasmi, I (Bentivoglio), 12

Fantesca, La (Parabosco), 49

Fantesca, La (Porta, Giovanni Battista della), 54 Farfalla (Lo Stechito da Siena), 62 
Favola d'Adone, La (Dolce, Lodovico), 27

Fedele, Il (Pasqualigo), 50

Fedeltà ingegnosa (Benedetto), 12

Fedra (Bozza), 14

Figliuolo prodigo, Il (Moro), 47

Filarmindo (Campeggi), 17

Filastoppa (Lo Strafalcione), 62

Filli di Sciro (Bonarelli della Rovere, Guidubaldo),

13

Filosofo, $I l$ (Aretino), 8

Fingere per vivere, Il (Tauro), 63

Finta fiammetta, La (Contarini), 24

Finta schiavetta, La (Moderati), 46

Finto paggio, overo amare, e non sapere chi, $I l$

(Stramboli), 62

Fiorina (Ruzzante), 57

Fiorina, La (Calmo), 16

Flaminio (Baroncini), 9

Flamminia prudente (Chaggio), 21

Flavia, La (Bellausa), 11

Flerida gelosa (Manzini), 44

Flora, La (Alamanni), 5

Flori (Campiglia), 17

Floria, La (Arsiccio Intronato), 9

Florinda (Settizonio), 60

Formicone (Filippo), 30

Fortuna (Bientina), 12

Fortunia, La (Il Desioso . . .), 26

Fortunio (Giusti), 34

Forza del fato, La (Cicognini), 22 
Forza dell'amicitia, La (Cicognini), 22

Forza del innocenza, La (Cicognini), 22

Frutti d'amore (Lauro), 40

Furto, Il (Ambra), 6

Galatea, La (Torelli, Pomponio), 64

Gaudio (Notturno Napolitano), 47

Gelopea (Chiabrera), 21

Gelosi, I (Gabiani), 31

Gelosia, La (Franceschi), 31

Gelosia, La (Grazzini) , 35

Gelosie fortunate del prencipe Rodrigo, Le (Cicognini), 21

Geloso, Il (Bentivoglio), 12

Generosità d'amore (Gnavio), 34

Ghirlanda, La (Anonymous), 1

Giardiniera (Moderati), 46

Giocasta (Dolce, Lodovico), 27

Giorgio (Bartolommei Smeducci), 10

Giosef filiuolo di Giacob (Martinelli), 44

Gismonda, La (Razzi), 55

Giustina, La (Morone), 47

Giustina Reina di Padoua (Cortesi), 25

Giustina vergine, e martire santissima (Liviera), 41

Giusto inganno, Il (Il Desioso...), 26

Glorie e gli amore di Alessandro Magno, e di Rossane, $L e$ (Cicognini), 22

Gostanza, La (Razzi), 55

Granchio, $I l$ (Salviati), 58

Gratiana (L'Infiammato). See under Anonymous Plays, 1 
Hadriana, La (Groto), 36

Hecuba, La (Dolce, Lodovico), 27

Hermafrodito, L' (Parabosco), 49, 50

Hidalba (Veniero), 66

Hipocrito, L' (Aretino), 7

Honesta schiava (Pico), 52

Honorata, L' (Cicognini) , 22

Hortensio, L' (Piccolomini). See under Anonymous Plays, 1

Idropica, La (Guarini, Giovanni Battista), 37

Ifigenia (Dolce, Lodovico), 27

Imenei ravvivati, $G l^{\prime}$ (Brina), 14

Imeneo, L' (Bonarelli della Rovere, Prospero), 13

Incantesimi, Gl' (Cecchi), 19, 20

Incendio, Lo (Loredano), 41

Ingannati, $G l$ ': comedia del Sacrificio degli Intronati... (Anonymous), 2

Ingannati, $G l$ ': commedia (Anonymous), 2

Inganni, Gl' (Secchi), 59

Inganni villaneschi, Gl' (Il Desioso . . .), 26

Ingiusti sdegni, Gl' (Pino), 52

Ingratitudine, La ([Ottonaio], Giovanni Battista dell'), 49

Innocente fanciulla, $L^{\prime}$ (Gabrielli, Gabriello), 32

Interesse, $L^{\prime}$ (Secchi), 59

Intricati, $G l^{\prime}$ (Pasqualigo, Aluise [Luigi]), 50

Intrichi d'amore (Tasso), 63

Intrighi amorosi, Gl' (Il Desioso ...), 26

Inuentione della croce di Giesu Christo, La (Poggi), 53 
Ioseph (Collenucci[o]), 23

Isabella (Bartolommei Smeducci), 10

Isabella, overo la donna più costante, L' (Tauro), 63

Isach, Lo (Groto), 36

Isifile (Mondella), 46

Isola d'Alcina, L' (Testi), 63

Ladro, Il (Comparini), 24

Ladro Cacco, Il (Il Desioso ... ), 26

Lamento di ser Cofaccia Buffone, Il (Boiardo), 13

Lamento pietoso del disgratiato Glonico . . . , Il ([Castalio, Sabba]), 18

Lazaro ricco: e di Lazaro pouero, La rappresentazione di (Anonymous), 3

Legge d'amore, La (Guerrini), 38

Lena, La (Ariosto), 8

Leonida, La (Ghirardi), 33

Lettere di cambio, Le ([Claudi, Francesco]), 23

Liberatione d'Amore (Il Desioso ... ), 26

Libero Arbitrio (Negro, Francesco, Bassanese), 47

Libro, Il (Selva), 59

Lilia: commedia pastorale (Anonymous), 2

Lilia: egloga pastorale (Anonymous), 2

Lite amorosa (Contrini), 24

Lucidi, I (Firenzuola), 30

Maga con Fritellino mago a caso, La (Dattomo), 25

Maga fulminata, $\mathrm{La}$ (Ferrari), 29

Magico legato, $I l$ (Benedetti), 12 
Magrino (Campani), 17

Malandrina, La (Loredano), 42

Malfatto (Anonymous), 2

Mal marito, Il (Persio), 51

Mandragola, La (Machiavelli), 43

Marescalco, Il (Aretino), 7

Margherita d'Antiochia, La (Quaretta), 54

Mariene ouero il maggior mostro del mondo, La (Cicognini), 23

Marinaio, Il (Parabosco), 49, 50

Maritaggio delle muse, Il (Riccio), 55

Marito, Il (Dolce, Lodovico) , 27, 28

Marito delle due moglie, Il (Cicognini), 23

Martirio di Santa Giuliana da Nicomedia, Il (Seragone), 60

Mascarate, et capricci dilettevoli . . . (Veraldo), 66

Mathilda, La (Guidozzo), 38

Matrigna, La (Loredano), 42

Medea (in Le tragedie di Seneca ...) (Dolce, Lodovico), 28

Medea (Galladei), 32

Medea, La (Dolce, Lodovico) , 27, 28

Medea essule, La (Zoppio), 68

Medico, Il (Castellini), 19

Medoro rappresentato in musica ..., Il (Salvadori), 58

Meganira (Chiabrera), 21

Megliacci, I (Podiani, Mario), 53

Merope, La (Torelli, Pomponio), 64

Mestola (Lanci), 39

Miracolo d'amore (Guazzo), 38 
Miracolo della Sacra Vergine Santa Caterina da Siena, Rappresentatione spirituale del (Il Malatesta), 43

Moglie, La (Cecchi), 19, 20

Moglie costante, La (Anonymous), 2

Moglie di quattro mariti, La (Cicognini), 23

Mora, La (Calderari), 16

Morti vivi, I (Oddi), 48

Moschetta (Ruzzante), 57

Mostro, Il (Anonymous), 2

Motti di Fortuna (Maniscalco), 44

Nascimento di Venitia (Cremonini), 25

Nativita di N.S. Giesu Cristo, La (Cornacchini), 24

Naue, La (Scardova), 59

Negromante, Il (Ariosto), 8

Niccolosa, La (Lanci), 39

Nice, $L a$ (Contile), 24

Nina (Pescatore), 51

Ninfa avara, $L a$ (Ferrari), 29

Ninfa cacciatrice, La (Bartolaia), 10

Notte, La (Parabosco), 49, 50

Noze de Psyche e Cupidine (Carretto), 18

Nozze d'Antilesina, Le (Pastor Monopolitano?), 50

Nozze di Hadriana, Le (Anonymous), 3

Nozze finte, Le (Micalori), 46

Nuove ottave alla Ciciliana ... (Anonymous), 3 .

See also Ottave 
Olivetta (Lanci), 39

Orbecche (Giraldi Cinthio), 33

Ortigia, $L^{\prime}$ (Viviani), 67

Ottave alla Siciliana (Anonymous), 2. See also

Nuove ottave

Ottauia furiosa (Marzi), 45

Ottauiano Imperadore, La rappresentatione e festa di (Anonymous), 4

Pace, La (Negro, Marin), 47

Panecchio (Il Fumoso della Congrega de' Rozzi), 31

Parentadi, I (Grazzini), 35

Pastor fido, Il (Guarini, Giovanni Battista), 37

Pastor regio, Il (Ferrari), 29

Pazzia, La (Sicinio) , 60

Pazzia politica di Roberto Rè di Sicilia, La (Tirinto), 64

Pazzie giovenili, Le (Gattici), 32

Pedante, El (Belo), 11

Pellagrilli (Lo Strafalcione), 62

Pellegrina, La (Bargagli), 9

Pellegrino, Il (Comparini), 24

Pellegrino, Il (Parabosco) , 49, 50

Pentesilea, La (Bracciolini) , 14

Pentimento amoroso, Il (Groto), 36

Perla (Balsamino), 9

Personaggi finti (Stanchi), 61

Pescara, La (Contile), 24

Pescatore (Roncaglia, Marcello), 56

Philostrato e Pamphila (Pistoia, Antonio da), 52 
Pidinzuolo (Anonymous), 3

Pieta d'amore (Maniscalco), 44

Pieta d'amore (Roncaglia, Marcello), 56

Piglia il peggio (Roncaglia, Giovanni), 56

Pinzochera, La (Grazzini), 35

Piovana (Ruzzante), 57, 58

Pippa, La (Anonymous), 3

Poesia maritata, La (Riccio), 55

Polietto (Bartolommei Smeducci), 10

Polifila (Gelli). See under Anonymous Plays, 3

Pomona (Alticotio), 6

Pompe funebri, ouero Aminta, e Clori (Cremonini), 25

Portia, La (Galanni), 32

Potione, La (Calmo), 16

Prencipe giardiniera, Il (Ferrari), 29

Prencipe Tigridoro, Il (Miari), 46

Prigione d'amore (Oddi), 48

Profezia (Fra Baseo), 10

Progne (Domenichi; from Latin of Corraro), 28

Progne, La (Parabosco), 50

Prova amorosa, La (Cesana), 20

Quando sta' peggio sta' meglio (Boccabadati), 13 Quindeci misterij del Santiss. Rosario, Rappresentationi sacre delli (Felisi), 29

Ragazzo, Il (Dolce, Lodovico), 27

Rapimento di Cefalo . . . , Il (Chiabrera), 21

Rappresentatione. See under first principal word of title 
Re Artemidoro, Il (Panciatichi), 49

Regina Ilidia, La (Vinta) , 67

Re Torrismondo, Il (Tasso), 63

Rhodiana (Calmo), 16. See also under Ruzzante, 57, 58

Rodopeia (Verlatto), 67

Roggiero sovrano della Calabria, Il (Grassi), 35

Romilda (Cesari), 21

Rosa, La (Cortese, Giulio Cesare), 25

Rosario trionfante, Il (Cini, Giacinto), 23

Rosaura, La (Stanchi), 61

Roselmina (Settizonio), 60

Rosmunda (Rucellai), 56

Rouinazo (Anonymous), 4

Ruffiano, Il (Dolce, Lodovico), 27

Sacrificio, Il (Beccari), 11

Saltuzza, Il (Calmo), 16

Sansone, La rappresentazione di (Roselli), 56

Santa Agata Vergine, e Martire, La rappresentazione di (Anonymous), 4

Santa Agnesa (Lottini) , 42

Santa Barbara, La devotissima rappresentatione di (Anonymous), 4

S. Cecelia, Rappresentatione di (Spezzani), 61

Santa Maria Egizziaca (Cicognini), 23

S. Grisante e Daria, Rappresentazione di (Anonymous), 4

Santi innocenti, I (Porta, Malatesta), 54

Santo Alesso, La rappresentazione di (Anonymous), 4 
S. Antonio abate..., La rapresentatione di (Anonymous), 4

S. Bastiano tratta dalla sua vita, Rappresentazione di (Lanci), 39

San Francesco, La rappresentazione di (Anonymous), 4

Santo Giorgio, cavaliere di Christo, La rappresentatione di (Anonymous), 4

San Giouanni Batista quando andò nel diserta, La rappresentazione di (Belcari), 11

San Lorenzo (Lottini), 42

Santo Panuntio Eremita, La rappresentatione di (Belcari), 11

Sarcodinamia, ciò̀ La possanza della carne (Glissenti), 34

Satiro, Il (Avanzi), 9

Sauina: egloga a la Martorella (Legacci), 40

Sauina: egloga rusticale (Legacci), 40

Sbratta, Lo (Pino), 52

Scambi, Gli (Aperto Accademico Intronato), 7

Scanniccio (Roncaglia, Giovanni), 56

Schiava, La (Calderari), 16

Schiaui d'amore, Gli (Podiani, Francesco), 53

Schiavo, Lo (Rettori), 55

Scilla (Cesari), 21

Scolastica, La (Ariosto), 8

Selene (Giraldi Cinthio), 33

Semiramis, La (Manfredi) , 44

Senafila (Il Desioso...), 26

Sensale, Il (Mercati), 45

Sergio, $I l$ (Fenarolo), 29 
Servigiale, Il (Cecchi), 20

Servo fidele, Il (Lunardi), 42

Sette beati fondatori della religione de' Servi (Lottini), 42

Sibilla, La (Grazzini) , 36

Sogno, Il (Guicciardi), 38

Solfinello (Legacci), 40

Solimano, Il (Bonarelli della Rovere, Prospero), 13

Sophonisba, La (Carretto), 18

Sophonisba, La (Trissino), 65

Soppositi, Gli (Ariosto), 8

Sorella, La (Porta, Giovanni Battista della), 54

Sosanna (Sacco), 58

Spagnolas, La (Calmo), 16

Spedale, Lo (Abati), 5

Speranza, La (Bartolucci), 10

Spina, La (Salviati) , 58

Spiritata, La (Grazzini), 36

Spirito, Lo (Cecchi), 19

Sponsalitio (Guarini, Alfonso di Battista), 36

Sporta, La (Gelli), 33

Staffle per gl'abusi d'hoggidi, Lo (Vallacca da Sarope), 66

Stiaua, La (Cecchi) , 19, 20

Straccioni, Gli (Caro), 18

Strascino (Campani), 17

Strega, La (Grazzini), 36

Successi d'amore, I (Ruschi), 57

Suocera, La (Varchi), 66 
Suppositi, I (Ariosto), 8

Susanna, La (Memmolo) , 45

Susanna, La rappresentatione e historia di (Anonymous), 4

Talanta, La (Aretino), 7

Tancia, La (Buonarotti), 15

Tancredi, Il (Campeggi), 17

Tancredi, Il (Torelli, Pomponio), 64

Tempio de amore (Carretto), 18

Teodora (Bartolommei Smeducci), 10

Theodora, La (Maleguzzi) , 43

Thesoro, Il (Groto), 36

Thieste (Dolce, Lodovico), 27

Thieste (in Le tragedie di Seneca . . .) (Dolce, Lodovico), 28

Thyeste: tragedia tratta da Seneca (Dolce, Lodovico), 28

Timone (Boiardo), 13

Tirrhenia (Aragona), 7

Tita (Il Desioso...), 26

Tonio e Pippo contadini, e l'Hoste (Anonymous), 4

Torti amorosi, I (Castelletti), 19

Tradimento amoroso, Il (Maggi), 43

Trappolaria, La (Porta, Giovanni Battista della), 54

Trauaglio, Il (Il Fumoso della Congrega de' Rozzi), 31

Tre constanti, Le (Marliani), 44

Tre tiranni, I (Ricchi), 55

Trinozzia, La (Contile), 24 
Trinutia, La (Firenzuola), 30

Trionfatrice Christina, La (Licco) , 41

Trionfo di Cristo, nella domenica delle palme (Falteri), 29

Trionfo di Pan Dio de' Pastori (Lionardo detto

Mescolino), 41

Tullia feroce (Cresci), 25

Turca, La (Loredano), 42

Turca, La (Porta, Giovanni Battista della), 54

Uccellatoio, L' (Sogliani), 60

Vaccaria (Ruzzante), 57, 58

Vedoua, La (Buonaparte), 15

Velettaio (Massucci), 45

Viluppo, Il (Parabosco), 49, 50

Vincitrice Caterina, La (Il Vigoroso . . .), 67

Virginia (Accolti), 5

Vitio muliebre (Maniscalco), 44

Zannin da Bologna (Anonymous), 4

Zecca, La. See Cecca 



\section{INDEX OF INTERMEDII}

Brief dramatic or musical entertainments bound with, included in, or written for the following plays.

Alceo (1614 ed.) (Ongaro), intramezzi of Guarini, 48

Cangenia, La (Poggi), 5 intermedii, 53

Cecaria. ... Aggiontoui un bellissimo lamento del Geloso con la Luminaria (Epicuro), 28

Commodo, Il (Landi), 5 intermedii, 39

Corsaro Arimante, Il. With Glauco schernito ... (Aleardi), 4 intermedii, 5-6

Esaltazione della croce, $L^{\prime}$ (Cecchi), 6 intermedii, 20

Giustina, La (Morone), 4 "tramezi spirituale," 47 Granchio, Il (Salviati), intermedii, 58

Honesta schiava (Pico, Girolamo), 5 intermedii of Giovanni Francesco Pico, 52

Intermedi rappresentati nella Finta Fiametta in Padoa (Contarini), 4 intermedii, 24

Intramezzi d'Erminia, tratti dalla Gerusalemme del Signor Torquato Tasso (Tortoletti), 5 intramezzi, 65 
Intrichi d'amore (Tasso), 5 intermedii of Giovanni Antonio Liberati, 63

Inuentione della croce di Giesu Christo, La (Poggi), 5 intermediary madrigals, 53

Maritaggio delle muse, $I l$ (Riccio), 4 intermedii in Italian and Latin, 55

Prosperpina rapita (Intermedio) (Ferrari), 30

S. Cecelia, Rappresentatione di (Spezzani), 5 intermedii, 61

Servigiale, $I l$ (Cecchi), 5 intermedii, 20

Trionfatrice Christina, La (Licco), 5 intermedii, 41 



SW

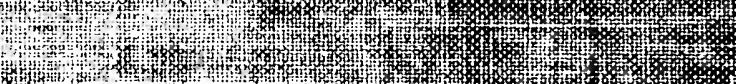

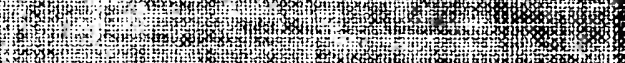

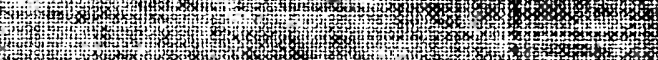

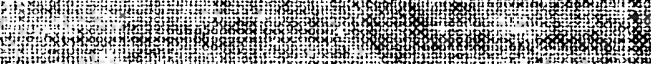

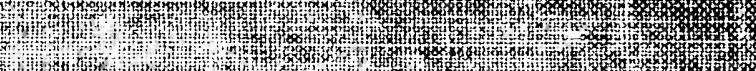

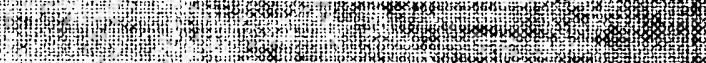

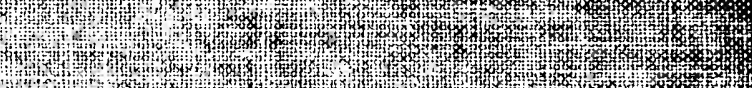

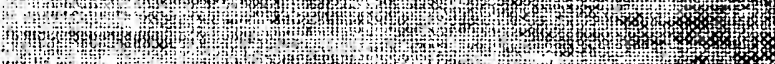

(7)

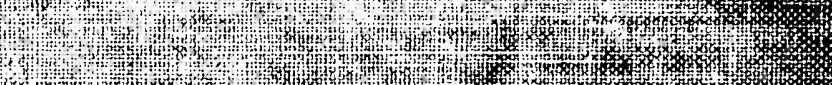

W

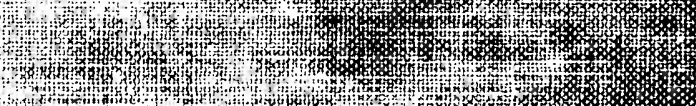

$\begin{array}{lll}7 & \end{array}$

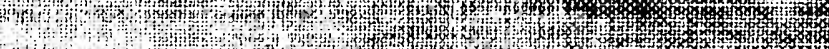

$\begin{array}{lll}4 & \end{array}$

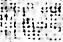

1
1

iqü:

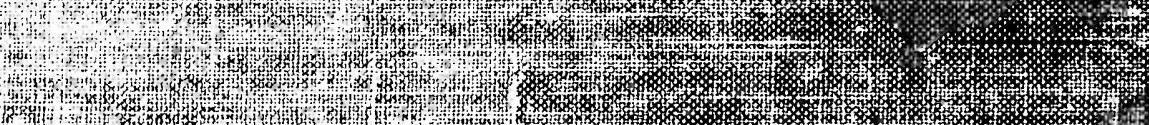

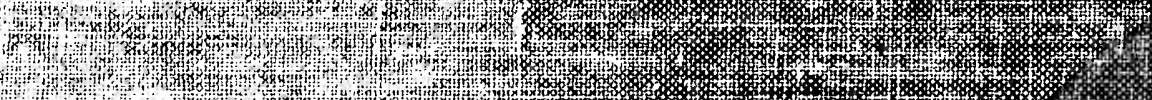

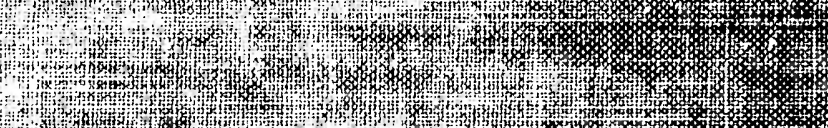

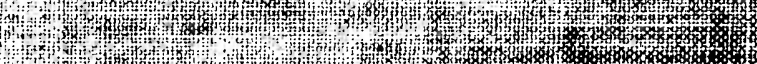

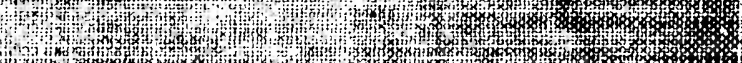

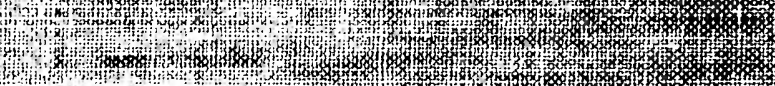

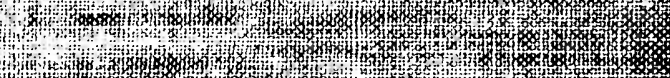

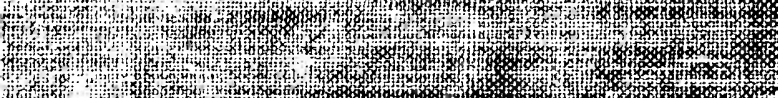

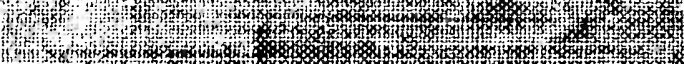

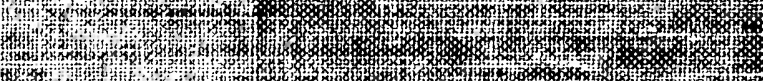

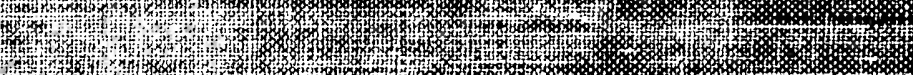

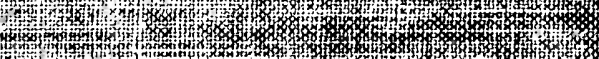

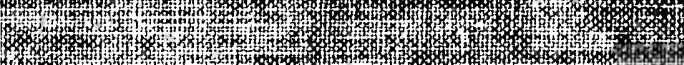

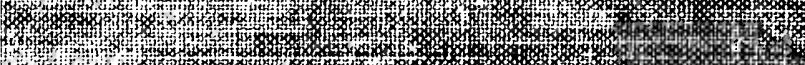

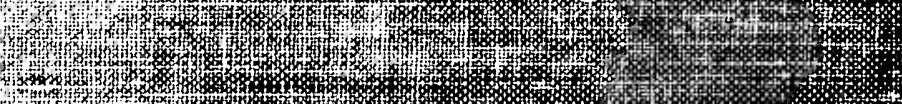

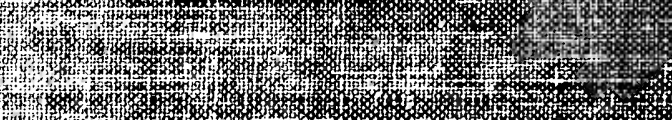

